\title{
The plant calcium-dependent protein kinase CPK3 phosphorylates REM1.3 to restrict viral infection
}

Artemis Perraki ${ }^{1,2,4^{*}}$, Julien Gronnier ${ }^{1,2^{*}}$, Paul Gouguet ${ }^{1,2}$, Marie Boudsocq ${ }^{5}$, Anne-Flore Deroubaix ${ }^{1,2}$, Vincent Simon $^{3}$, Sylvie German-Retana ${ }^{3}$, Cyril Zipfel ${ }^{4}$, Emmanuelle Bayer $^{1,2}$, Sébastien Mongrand ${ }^{1,2}$, Véronique Germain ${ }^{1,2}$

${ }^{1}$ CNRS, Laboratoire de Biogenèse Membranaire (LBM), UMR 5200, F-33000 Bordeaux, France

${ }^{2}$ Univ. Bordeaux, Laboratoire de Biogenèse Membranaire (LBM), UMR 5200, F-33000 Bordeaux, France

${ }^{3}$ Equipe de Virologie UMR BFP 1332 INRA, 33883 Villenave d'Ornon, France

${ }^{4}$ The Sainsbury Laboratory, Norwich Research Park, Norwich, NR4 7UH, United Kingdom.

5 Institute of Plant Sciences Paris Saclay (IPS2), CNRS, INRA, Université Paris-Sud, Université d'Evry, Université Paris-Saclay, Université Paris-Diderot, Sorbonne Paris-Cité, Bâtiment 630, Plateau du Moulon, 91192 Gif sur Yvette, France

Present addresses:

Artemis Perraki, Department of Plant Sciences, University of Cambridge, Downing Street, Cambridge CB2 3EA.

Julien Gronnier, The Sainsbury Laboratory, Norwich Research Park, Norwich, NR4 7UH, United Kingdom.

*, AP and JG contribute equally to this work and must be considered as co-first authors

Corresponding author: Sébastien MONGRAND, Laboratoire de Biogenèse Membranaire UMR 5200 CNRS - Université de Bordeaux. Bâtiment A3 - INRA Bordeaux Aquitaine. 71 Avenue Edouard Bourlaux CS 20032 F-33140 Villenave d'Ornon FRANCE. Tel: +33 (0)5 57 1225 37; Fax: +33 (0)5 571226 44. e-mail: sebastien.mongrand@u-bordeaux.fr 


\begin{abstract}
Plants respond to pathogens through dynamic regulation of plasma membrane-bound signaling pathways. To date, how the plant plasma membrane is involved in responses to viruses is mostly unknown. Here, we show that plant cells sense the Potato virus X (PVX) COAT PROTEIN and TRIPLE GENE BLOCK 1 proteins and subsequently trigger the activation of a membrane-bound calcium-dependent kinase. We show that the Arabidopsis thaliana CALCIUM-DEPENDENT PROTEIN KINASE 3-interacts with group 1 REMORINs in vivo, phosphorylates the intrinsically disordered N-terminal domain of the Group 1 REMORIN REM1.3, and restricts PVX cell-to-cell movement. REM1.3's phospho-status defines its plasma membrane nanodomain organization and is crucial for REM1.3-dependent restriction of PVX cell-to-cell movement by regulation of callose deposition at plasmodesmata. This study unveils plasma membrane nanodomain-associated molecular events underlying the plant immune response to viruses.
\end{abstract}




\section{Introduction}

The cell plasma membrane (PM) constitutes a regulatory hub for information processing where intrinsic and extrinsic signals are perceived, integrated and relayed 1. PM proteins and lipids dynamically associate with each other, creating specialized sub-compartment that regulate the cellular responses in space and time ${ }^{2-4}$. In animal, modeling of a PM-bound receptor and its downstream interactor before and after ligand perception, suggests that PM-partitioning into nanodomains improves the reliability of cell signaling ${ }^{5}$. In plants, the immune and growth receptors FLS2 and BRI1 are divided into context-specific nanodomains, allowing them to regulate distinct signaling pools at PM, despite sharing several signaling components ${ }^{6}$. The REMORIN (REM) family is probably one of the best-characterized PM nanodomain-associated proteins in plants ${ }^{6-11}$. The association of REMs to the PM is mediated by a short sequence at the extremity of the C-terminus of the protein, called REM-CA (REMORIN C-terminal Anchor) ${ }^{12}{ }^{13}$. The REM C-terminal domain, contain a predicted coiled-coil (residues 117-152) which is thought to regulate REM oligomerization 10, 13, 14 and may be involved in regulating REM spatial organization at the PM. Members of the REM family have been associated with plant responses to biotic ${ }^{8,15,16}$, abiotic stress ${ }^{17}{ }^{18}$ and developmental clues ${ }^{11}$ and current view suggest they could regulate signaling events through nanodomain association. However, the molecular mechanisms leading to REM downstream events remain elusive. Several REM proteins have been identified as components of the plasmodesmata-plasma membrane subcompartment (PD-PM) ${ }^{7,19,20}$. PD are membranous nanopores, crossing the plant cell wall and enabling cytoplasmic, endoplasmic reticulum and PM continuity between adjacent cells. They regulate the intercellular transport of proteins and small molecules during development and defense ${ }^{21}$ 22. The PD-PM is a particular subcompartment of the PM which displays a unique molecular composition and is enriched in sterols ${ }^{23}$. The movement of macromolecules through PD can be tightly controlled through modulation of the PD size-exclusion limit (SEL) via hypo- or hyper-accumulation of callose at the PD neck region ${ }^{24-26}$. Overexpression of GRAIN SETTING DEFECT 1 (GSD1), encoding a phylogenetic group 6 REM protein from rice, restricts PD aperture and transport of photoassimilates ${ }^{20}$.

$\mathrm{PD}$ are also the only route available for plant viruses to spread from cell-to-cell. Potato virus $X$ (PVX) promotes its cell-to-cell movement via modification of PD permeability ${ }^{27}$ through the action of TRIPLE GENE BLOCK PROTEIN 1 (TGBp1) and callose removal ${ }^{28}$. Overexpression of StREM1.3 (Solanum tuberosum REM from group $1 \mathrm{~b}$, homolog $3{ }^{29}$ ), further referred as REM1.3, hampers TGBp1 ability to increase PD permeability ${ }^{30}$. How is REM1.3 obstructing TGBp1 action is still unknown but its lateral organization into nanodomains at the PM is directly linked with REM1.3 ability to restrict PVX movement and regulate PD conductance ${ }^{31}$.

REM1.3 was the first REM family member discovered and initially described as a protein phosphorylated upon treatment with oligogalacturonides, which are plant cell wall components and elicitors of plant defense ${ }^{32,33}$. The biological relevance of REM phosphorylation is not known, yet 
reports of different REM phospho-statuses suggest that the activity of these proteins could be regulated by phosphorylation during plant-microbe interactions ${ }^{14,15,34,35}$.

In the present paper, we show that phosphorylation of REM dictates its membrane dynamic and antiviral defense by the reduction of PD permeability. Our data points toward a model whereby viral proteins such as the Coat Protein (CP), TGBp1 from PVX and 30K proteins from Tobacco mosaic virus (TMV) elicit the activation of protein kinase(s), which in turn phosphorylate(s) REM1.3 at its Nterminal domain. In turn, REM1.3's phospho-status regulates its spatial-temporal organization at the PM and association with PD. The latter is associated with PD closure by induction of PD callose deposition at PD and restriction of viral cell-to-cell movement. Last, we further provide evidence that the membrane bound Arabidopsis thaliana CALCIUM-DEPENDENT PROTEIN KINASE 3 (CPK3) interacts with the taxonomic group $1 \mathrm{~b}$ REMs ${ }^{7}$ in vivo, phosphorylates REM1.3 in vitro and restricts PVX propagation in a REM-dependent manner. Collectively, this study brings valuable information about the involvement of PM nanodomains dynamics during the establishment of membrane-bound signaling processes. 


\section{Results}

PVX triggers changes in REM1.3 membrane dynamic behavior and REM1.3 association with plasmodesmata

Group 1 and group 6 REM have been described as PD-localized protein regulating PD size-exclusion limit ${ }^{7} 20{ }^{30}$. REM1.3 plays a role in restricting PVX passage through the PD channels ${ }^{730}$, whereas in the same time PVX movement proteins promote opening of $\mathrm{PD}{ }^{36}$. To study the potential function of REM1.3 at PD in response to PVX infection, we surveyed simultaneously PD callose content and REM1.3 PD localization in healthy or PVX-infected $N$. benthamiana transgenic lines overexpressing YFP-REM1.3 ${ }^{37}$ (Fig EV1). Our analysis firstly showed a significant increase in callose deposition in PVX-infected cells (Fig 1A,B). This finding suggests the recognition of PVX-encoded elicitor and the mobilization of a plant defense response leading to an increase of callose accumulation at PD pit fields.

Since protein activation is often linked to changes in subcellular localization, even to specific PM subcompartments 2, 38, we next examined whether PVX infection triggers changes in REM1.3 PD localization. Calculation of the PD index (ratio between fluorescence intensity of YFP-REM1.3 at the aniline-labeled PD pit field and fluorescence at the PM around the pit field ${ }^{25}$, Fig EV1) surprisingly showed that despite its direct role on PD regulation YFP-REM1.3 is not enriched in the PD region of healthy $N$. benthamiana epidermal cells. Interestingly, we however reproducibly observed a slight increase of YFP-REM1.3 PD index upon PVX infection (Fig 1A,B) suggesting that PVX perception modulates REM1.3 localization and association with the PD pit fields.

To gain further insights into REM1.3 dynamic localization at the PM upon PVX infection, we applied single-particle tracking Photoactivated Localization Microscopy in Variable Angle Epifluorescence Microscopy mode (spt-PALM VAEM) in living $N$. benthamiana cells in absence or presence of PVX. By this approach, we recently studied the protein organization and mobility parameters of single EOSREM1.3 molecules in non-infected conditions and found that EOS-REM1.3 displays a mostly immobile and confined PM localization pattern, as commonly observed for plant membraneassociated proteins (Fig 1C-E) ${ }^{39}{ }^{31}$. Reminiscent of these data, previous studies using different techniques described REM-associated PM domains to be predominantly stable to lateral diffusion ${ }^{31}$, 39, 40. Analysis of PVX-infected cells demonstrated an increase of EOS-REM1.3 diffusion coefficient (D) and mean square displacement (MSD), reflecting an increase of REM1.3 mobility (Fig 1C-E). We next compared the supra-molecular organization of EOS-REM1.3 by Voronoï tessellation of live PALM data ${ }^{31} 41$ in mock and PVX infected conditions (Fig 1F). Computation of EOS-REM1.3 single molecule organization features demonstrated a modulation of REM1.3 nanodomain-organization upon PVX infection (Fig 1G). Following PVX infection, the EOS-REM1.3-formed nanodomains are bigger in size, and there is a slight decrease of the proportion of molecules that localizes into nanodomains as well as a significant decrease in the number of nanodomains formed. Overall, in both conditions, EOS-REM1.3 nanodomains represented similar proportions of the total PM surface. Additionally, a 
significant decrease in the localization density (number of molecules observed per $\mu \mathrm{m}^{2}$ per s), shows that upon PVX infection, there is less protein at PM level, which could reflect relocalization of molecules to other compartments. Overall, the changes of REM1.3 distribution at the PM under PVX infection i.e. enrichment of YFP-REM1.3 in the pit field regions, the increase of REM1.3's mobility and the modulation of REM1.3 nanodomain organization, suggest that the plant cell might modulate PD-PM nanodomain dynamics to circumvent PVX infection.

\section{Perception of PVX proteins by plant cells leads to the activation of kinase(s) phosphorylating REM1.3}

REM1.3 overexpression was shown to restrict PVX spreading in both Solanum lycopersicum ${ }^{7}$ and Nicotiana benthamiana ${ }^{30}, 31$. As REM1.3 was originally discovered as a PM-associated phosphorylated protein ${ }^{33}$, we asked whether we could detect kinase activity in $N$. benthamiana extracts leading to REM1.3 phosphorylation and whether this activity was induced by PVX presence in the cells. Equal protein amounts of microsomal and soluble extracts from $N$. benthamiana leaves were used as a kinase source to phosphorylate affinity-purified full-length 6His-REM1.3 in an in vitro kinase assay in the presence of ATP $\left[\gamma^{3}{ }^{33} \mathrm{P}\right]$. Autoradiography revealed the presence of a clear band corresponding to a phosphorylated form of 6His-REM1.3 by kinase(s) present in the microsomal fraction (Fig 2A). The intensity of this band was reduced and completely abolished by competition with cold ATP, but not cold AMP, indicating that this was a valid experimental set-up to study a genuine transphosphorylation event (Fig EV2A). Phosphorylation of 6His-REM1.3 was almost not detectable in soluble fractions, representing cytosolic kinases (Fig 2A).

In silico analysis predicted phosphorylation sites throughout REM1.3 sequence (Diphos, DEPP and NETPHOS prediction software). In agreement with the location of the sites presenting the highest phosphorylation potential, we experimentally found that REM1.3 was phosphorylated in its Nterminal domain (residues 1-116, hereby 6His:REM1.3 $3^{\mathrm{N}}$ ) whereas the C-terminal domain (residues 117-198, hereby 6His:REM1.3 ${ }^{\mathrm{C}}$ ) did not present any detectable phosphorylation (Fig EV2B,C). We next tested whether PVX activates the membrane-associated kinase(s) that phosphorylate(s) REM1.3. Our results unveiled that microsomal and PM fractions extracted from symptomatic PVX-infected leaves promoted higher levels of 6His-REM1.3 phosphorylation compared to extracts from noninfected plants (Fig 2B,C).

Studies have shown that functionally different viral components, such as virus-encoded proteins and double stranded RNA, can trigger plant defense responses $424344,4546,47$. We examined whether the PVX genome in its free form was an eliciting signal for kinase activation and found that the addition of total RNAs extracted from PVX-infected plants in the kinase reaction mix did not alter the levels of 6His-REM1.3 phosphorylation (Fig 2D). We then examined whether the sole expression of individual viral movement proteins was sufficient to trigger REM1.3 phosphorylation. Importantly, our results demonstrated that the expression of TGBp1 and Coat Protein (CP) fused to GFP triggered the 
strongest levels of 6 His-REM1. $3^{\mathrm{N}}$ phosphorylation to the same extent as the full PVX-GFP construct (Fig 2E). TGBp2 and TGBp3 proteins also induced 6 His-REM1.3 ${ }^{\mathrm{N}}$ phosphorylation but to a lesser extent than TGBp1, CP, or full PVX-GFP. Quantification of three independent biological experiments shows that TGBp2 and TGBp3-induced 6His-REM1. ${ }^{\mathrm{N}}$ phosphorylation at levels comparable to the control mock experiments (Fig 2E). Expression of a TGBp1-deleted version of PVX (PVX $\Delta$ TGBp1) decreased 6His-REM1.3 phosphorylation levels compared to wild-type PVX extracts, but still to a greater level than GFP alone (Fig EV2D). Infiltration of the empty Agrobacterium strain alone induced stronger phosphorylation on 6 His-REM1. $3^{\mathrm{N}}$ in N. benthamiana epidermal cells than the water control (Fig 2E) supporting that REM can be differentially phosphorylated during plant-microbe interactions $^{8}$. Interestingly, expression of the 30K-RFP protein from Tobacco mosaic virus (TMV) also induced REM phosphorylation (Fig EV2E), suggesting a broader role of REM-mediated plant response to different genera of viruses. In good agreement with this, REM1.3 was shown to interfere with the ability of both PVX-TGBp1 and TMV-30K to increase PD permeability ${ }^{30}$. Concomitantly, overexpression of REM1.3 was found to restrict TMV-GFP cell-to-cell movement in N. benthamiana epidermal cells (Fig EV3A).

Collectively these results suggest that the triggering of REM1.3 phosphorylation by the perception of viral proteins by plant cells might regulate REM1.3 function in PD permeability regulation.

\section{Phosphorylation of REM1.3 on critical residues of its $\mathbf{N}$-terminal domain regulates its function in restricting PVX spreading via PD aperture modulation}

Since differential phosphorylation of REM occurs upon PVX infection, we next aimed to functionally characterize the importance of REM1.3 phosphorylation for the regulation of PVX cell-to-cell movement. Despite our efforts, the identification of in vivo phosphorylation sites of REM1.3 appeared technically challenging and remained unsuccessful to this day. In silico predictions and in vitro kinase assays however showed that REM1.3 $3^{\mathrm{N}}$ displays regions of intrinsic disorder and presents the highest potential of phosphorylation (Fig 2 C,E, and Fig 3A). For functional characterization, we selected the three putative phosphorylation Ser/Thr sites present in REM1.3 ${ }^{\mathrm{N}}$, namely S74, T86 and S91, that presented high scores of phosphorylation prediction in intrinsic disorder regions (Fig 3A). S74 and S91 are conserved across the phylogenetic group $1 \mathrm{~b}$ of REM proteins, suggesting functional redundancy (Fig EV5A) ${ }^{29,48}$. S74 and S91 were the analogous residues identified as phosphorylated in vivo in the group 1b REM AtREM1.3 (At2g45820) of Arabidopsis thaliana (hereby Arabidopsis) in a stimuli-dependent manner ${ }^{34,35,48}$. Biochemical analysis showed that alpha-1,4-poly-D-galacturonic acid (PGA)-induced phosphorylation of StREM1.3 occurs on T32, S74 and T86 ${ }^{49}$. T86 is not conserved in Arabidopsis but it is conserved in Solanaceae REM proteins, such as in N. benthamiana (Fig EV5A). By an in vitro kinase assay, we were able to demonstrate that S74, T86 and S91 are true phosphorylated residues of REM1.3, since substitution of S74, T86 and S91 to the non- 
phosphorylable Aspartic acid (D), generating the 6His-REM1.3 ${ }^{\mathrm{DDD}}$ mutant abolished REM phosphorylation by the PVX-activated kinase(s) (Fig 3B,C).

To functionally characterize the relevance of different REM1.3 phospho-statuses in the context of PVX-GFP propagation and PD-aperture regulation, we generated RFP- and YFP-fused REM1.3 mutants substituted at those sites either with Aspartic acid (REM1.3 ${ }^{\mathrm{DDD}}$ ) to mimic constitutive phosphorylation (hereby termed phosphomimetic mutant) or with Alanine (REM1.3 ${ }^{\mathrm{AAA}}$ ) representing null-phosphorylated state (hereby termed phosphodead mutant). Infection assays in N. benthamiana demonstrated that the phosphodead mutant lost ability to induce the restriction of PVX-GFP spreading, while the phosphomimetic mutant maintained this ability (Fig 3D). In good agreement with the TMV-30K-stimulated phosphorylation of REM1.3 presented in Fig EV2E, we confirmed that TMV-GFP propagation was also affected by the phospho-status of REM1.3 to the same extent as for PVX-GFP (Fig EV3A).

We then analyzed the capacity of REM1.3 phosphomutants to regulate PD aperture in the absence of viral infection. As previously described ${ }^{30,31}$, RFP-REM1.3 reduces the PD size-exclusion limit as measured by free GFP diffusion to neighboring cells (Fig 3E). Detailed phenotypic analysis of REM1.3 phosphorylation mutants demonstrated that the phosphomimetic mutant recapitulated REM1.3 activity towards PD-aperture regulation, while the phosphodead mutant did not (Fig 3E). Altogether, these results provide strong evidence that REM1.3's phosphorylation state at the evolutionarily conserved positions of S74, T86 and S91 is linked to its function in controlling viral infection and PD conductance.

\section{REM1.3 phospho-status modulates its dynamic lateral segregation in PM and PD sub- compartments}

Upon PVX infection we observed a modulation of REM1.3 PD-association and PM dynamics (Fig 1), linked to REM1.3 phosphorylation (Fig 2) that is required for REM1.3 function against PVX infection (Fig 3). Hence, we asked whether different REM1.3 phospho-statuses might regulate its lateral organization at the PM and PD compartments in the absence of PVX. We examined the enrichment of REM1.3 phosphomutants at the PD pit fields, previously calculated by the PD index (Fig 1A,B) and found that similarly to YFP-REM1.3, none of the phosphomutants appeared enriched at the pit field level (Fig 4A, C). The phosphodead mutant appeared statistically more excluded than YFP-REM1.3, whereas the phosphomimic mutant displayed an increase of its PD index (Fig 4C), reminiscent of the REM1.3 localization phenotype under PVX infection (Fig 1A,B). Importantly, REM1.3 phosphomutants's association with PD was directly correlated with callose content at PD (Fig 4B). These observations reinforced the hypothesis that REM1.3-mediated increase of callose levels at the PD is associated with a dynamic and phosphorylation dependent redistribution of REM1.3 to the PD surroundings. 
We examined by confocal microscopy the localization pattern of the phosphomutants fused to YFP. Both phosphodead (YFP-REM1.3 ${ }^{\mathrm{AAA}}$ ) and phosphomimetic (YFP-REM1.3 ${ }^{\mathrm{DDD}}$ ) mutants located exclusively at the PM, analogous to REM1.3 localization (Fig EV3B). We next used spt-PALM VAEM to characterize the localization and mobility behavior of the EOS-REM1.3 phosphomutants. The analysis of reconstructed trajectories and corresponding super-resolved localization maps indicated slight modifications of lateral mobility behavior between the phosphomutants (Fig 4 D, E). Quantification of the diffusion coefficient values (D) extracted for each individual molecule revealed that EOS-REM1.3 $3^{\mathrm{AAA}}$ displayed a more immobile behavior than EOS-REM1.3 ${ }^{\mathrm{DDD}}$ and EOS-REM1.3. Consistently, EOS-REM1.3 ${ }^{\text {DDD }}$ exhibited a higher mobility illustrated by higher diffusion coefficient and mean square displacement values (Fig 4 D, E). Analysis of the supra-molecular organization of the phosphomutants by Voronoï tessellation (Fig 4F) firstly showed that all mutants displayed similar nanodomain size and localization density compared to EOS-REM1.3 ${ }^{\mathrm{WT}}$. Compared to EOSREM1.3 ${ }^{\mathrm{AAA}}$, the EOS-REM1.3 ${ }^{\mathrm{DDD}}$ nanodomains occupied a smaller area of the total PM and their density in the PM plane appeared slightly reduced (Fig 4F,G). A higher number of nanodomains were formed with the EOS-REM1.3 $3^{\mathrm{AAA}}$ mutant. Hence, the phosphomimetic mutations favor a less confined and a more dynamic localization pattern of REM1.3 at the PM, reminiscent to the phenotype of EOS-REM1.3 ${ }^{\mathrm{WT}}$ in the context of PVX infection (Fig 1C,D). These results suggest that differential REM1.3 phosphorylation is involved in regulating REM1.3 mobility and PM domain organization and support the hypothesis that REM1.3 phosphorylation on S74, T86 and S91 reflects an 'active form' of the protein necessary for REM1.3-mediated defense signaling.

\section{AtCPK3 phosphorylates REM1.3}

To gain more insights into the signaling processes leading to REM1.3 phosphorylation, we aimed to biochemically characterize the kinase(s) involved in the phosphorylation of REM1.3. Previous evidence suggested that the kinase(s) phosphorylating REM1.3 are membrane-associated (Fig 2) ${ }^{33}$. We therefore biochemically analyzed the localization of the kinase(s) phosphorylating REM1.3. Plant material from healthy and PVX-GFP-infected leaves was cell-fractionated to obtain crude extracts, soluble and microsomal fractions ${ }^{50}$ to perform in vitro kinase assays on REM1.3 ${ }^{\mathrm{N}}$. Analysis confirmed a maximal kinase activity in purified microsomes (Fig 5A, 2A). Since a kinase in close proximity with its substrate would enhance reaction kinetics ${ }^{51}$ and signal fidelity ${ }^{52}$, and given that REM1.3 is enriched in detergent-resistant membranes (DRM) ${ }^{7}$, we investigated whether the kinase activity towards 6His-REM1.3 is enriched in this biochemical fraction. We included "control PM" (CPM) preparations, submitted to discontinuous sucrose gradients but in the absence of Triton-X100 treatments ${ }^{53}$. In vitro kinase assays on 6 His-REM $1.3^{\mathrm{N}}$ showed that the kinase activity in C-PM was 5 times inferior than in freshly purified PM not submitted to the sucrose gradient, suggesting that the kinase is not stable during the overnight purification procedure. Only half of the specific activity of 
the kinase was found in DRMs compared to the C-PM fraction, indicating that the kinase(s) phosphorylating REM1.3 is (are) only partially located in the DRM fraction (Fig 5B).

To gain more information concerning the biochemical characteristics of the kinase phosphorylating REM1.3, we analyzed its activity in the presence of known inhibitors. We firstly tested staurosporine, 54, 55 a general inhibitor that prevents ATP binding to kinases and found inhibition of REM1.3 phosphorylation starting at very low concentrations ( $30 \mathrm{nM})$ (Fig EV4A). We further tested the effect of poly-L-lysine, described to stimulate the activity of the CK2 kinases and inhibit several CDPK kinases ${ }^{56,57}$. No significant differences on REM1.3 phosphorylation levels were observed under increasing concentrations of poly-L-lysine (Fig EV4B). The addition of the wide range of Ser/Thr phosphatases inhibitor $\beta$-glycerophosphate $\left(\mathrm{BGP}^{58}\right.$ ) to the reaction mix did not alter the levels of phosphorylated 6His-REM1.3, indicating that the observed data was due to the activation of kinase activity by PVX rather than by inhibition of phosphatases (Fig EV4B). Competition assays in the presence of cold AMP and GTP showed that only cold ATP even at $2 \mathrm{mM}$ caused 20 -fold depletion in $\left[\gamma_{-}{ }^{33} \mathrm{P}\right]$ incorporation, suggesting that ATP is the major phosphoryl-donor for the kinase (Fig EV4B). Addition in the reaction mix of $0,2 \mathrm{mM}$ of EGTA, a chelator of $\mathrm{Ca}^{2+}$, strongly inhibited the kinase activity suggesting that the kinase(s) phosphorylating REM1.3 in healthy leaves is calcium sensitive (Fig EV4C). Calcium is a conserved second messenger in signal transduction during biotic and abiotic stress. In plants, kinases harboring different calcium sensitivities can perceive calcium variations and translate them into downstream signaling activation ${ }^{59}{ }^{60}$. To determine whether the PVX-activated kinase phosphorylating REM1.3 is sensitive to calcium regulation, in vitro kinase assays from microsomes of healthy and PVX-infected $N$. benthamiana leaves were assayed in the presence of free calcium $\left(\mathrm{Ca}^{2+}\right)$ concentrations ranging from $10 \mathrm{nM}$ to $0,1 \mathrm{mM}$. Figure $5 \mathrm{C}$ shows that the kinase(s) displays a high sensitivity to calcium with an optimal activity in the presence of $10 \mu \mathrm{M}$ of free $\mathrm{Ca}^{2+}$. At this concentration, a 5-fold increase of 6 His-REM $1.3^{\mathrm{N}}$ phosphorylation was observed in PVXinfected leaves (Fig 5C). These experiments allowed us to narrow-down the kinase(s) phosphorylating REM1.3 after PVX infection to the group of membrane-bound $\mathrm{Ca}^{2+}$-dependent protein kinases ${ }^{59}$. Plants possess three main families of calcium-regulated kinases: calmodulin-binding kinases (CBKs), calcineurin B-like-interacting protein kinases (CIPKs) and calcium-dependent protein kinases (CDPKs) ${ }^{58}$. CDPKs have the unique feature of calcium sensing and responding activities in one single polypeptide, best characterized in the model plant Arabidopsis thaliana ${ }^{59}$. Based on the measured calcium dose response (Fig 5C), we correlated the kinase phosphorylating REM1.3 in $N$. benthamiana with homologs of Arabidopsis thaliana subgroup II AtCPKs ${ }^{61}$, and we aimed to capitalize on the knowledge of Arabidopsis CPKs to test REM1.3 phosphorylation. Among the characterized members of subgroup II AtCPKs, we selected the Arabidopsis thaliana AtCPK3 as a good candidate to test its putative role in REM1.3 phosphorylation, since previous proteomics studies in Arabidopsis thaliana have identified both AtCPK3 and AtREM1.3 as being enriched in PM, PD 
and DRM fractions ${ }^{19,62}$. In addition, one study showed that AtREM1.3 from microsomal fractions is phosphorylated in vitro by AtCPK $3{ }^{63}$.

We therefore predicted that REM1.3 might share common functions with the evolutionarily conserved group 1b Arabidopsis REMs ${ }^{29}$. AtREM1.2 and AtREM1.3 are close homologs to REM1.3 and group $1 \mathrm{~N}$. benthamiana REMs (NbREMs) in term of protein sequence ${ }^{29,31}$ and they conserved at least the S74 and S91 phosphorylation sites 343548 (Fig EV5A). Using super-resolution microscopy, Demir et al. showed that -when co-expressed in Arabidopsis leaves- REM1.3 and AtREM1.3 co-localized in the same PM-nanodomains ${ }^{64}$. Importantly, transient expression of AtREM1.2 and AtREM1.3 in $N$. benthamiana epidermal cells impaired PVX-GFP cell-to-cell movement, as REM1.3 does (Fig EV5B), strengthening the hypothesis that the function of group 1 REMs might be conserved between homologs in different plant species ${ }^{31}$.

We assayed the in vitro phosphorylation activity of the affinity-purified AtCPK3-GST towards the 6 His-REM1.3, the 6 His-REM1. $3^{\mathrm{N}}$ and the 6 His-REM1. $3^{\mathrm{C}}$ as substrates. Similar to our previous results (Fig EV2B,C), AtCPK3-GST could phosphorylate strongly both 6His-REM1.3 and 6His-REM1.3 ${ }^{\mathrm{N}}$, but not 6 His-REM1.3 ${ }^{\mathrm{C}}$ (Fig 5D). Addition of $\mathrm{Ca}^{2+}$ is essential for a strong kinase activity as shown by both kinase auto-phosphorylation and trans-phosphorylation (Fig 5D). AtCPK3-GST specifically phosphorylated S74 T86 and S91 residues of REM1.3, since the phosphorylation was abolished in the phosphomimetic mutant 6His-REM1.3 ${ }^{\text {DDD }}$ (Fig 5E). These results suggest that AtCPK3 is a good candidate for group 1b REM phosphorylation and further support that the S74, T86, and S91 are the true phosphorylation sites of REM1.3 (Fig 3A, 5E).

\section{AtCPK3 interacts with group 1b REMs and restricts PVX propagation in a REM-dependent manner}

CPKs harbor a variable N-terminal domain, a Ser/Thr kinase domain, an auto-inhibitory junction region and a regulatory calmodulin-like domain. The calmodulin-like domain contains four EF-hand binding motifs that determine the sensitivity of each kinase to calcium ${ }^{65}{ }^{66}$. To investigate the role of AtCPK3 in REM-dependent signaling, we generated AtCPK3 mutants presenting altered kinase activities. Deletion of the inhibitory junction region and the regulatory calmodulin-like domain in CPKs creates a constitutive active kinase while mutation of the aspartic acid residue in the catalytic center 'DLK' motif of the kinase domain to an alanine (D202A) creates a catalytically inactive or 'dead' kinase ${ }^{59}$ (Fig 6A). We generated AtCPK3 full-length (AtCPK3), constitutive active (AtCPK3CA, 1-342) and kinase-dead (AtCPK3CA ${ }^{\mathrm{D} 202 \mathrm{~A}}$ ) constructs for transient protein expression (Fig 6A). We evaluated their catalytic activities by expressing them transiently in Arabidopsis mesophyll protoplasts and performing immunoprecipitation coupled to kinase assays using 6HisREM1.3 and histone as a generic substrate ${ }^{59}$. Autoradiography confirmed that in vivo purified AtCPK3CA-HA could trans-phosphorylate both 6His-REM1.3 and histone without the addition of calcium, while the point mutation D202A drastically abolished kinase activity (Fig EV6). 
We next examined the sub-cellular localization of both AtCPK3 and AtCPK3CA fused to YFP and found that both proteins disclosed a partial association with the PM, which was further confirmed by their presence, after cell fractionation, in the microsomal fraction at the expected molecular weight (Fig 6B) in good agreement with Mehlmer et al. ${ }^{63}$. We further used AtCPK3CA to test the interaction with group $1 \mathrm{~b}$ REMs. Bimolecular Fluorescence Complementation (BiFC) experiments showed that AtCPK3CA and REM1.3, REM1.3 $3^{\mathrm{AAA}}$ and REM1.3 ${ }^{\mathrm{DDD}}$ interact together at the level of the PM in planta. Importantly, we also confirmed the interaction of AtCPK3CA with homologous AtREM1.2 and AtREM1.3 (Fig 6C). REM1.3/REM1.3 interaction was used as a positive control, and AtCPK3CA /AtCPK3CA as a negative control.

We finally aimed to functionally characterize the AtCPK3- and REM1.3-mediated signaling in the context of PVX infection. Transient over-expression of AtCPK3-RFP alone induces a reduction of PVX-GFP infection foci, confirming that AtCPK3 is indeed important for antiviral responses in plant cells (Fig 6D). Expression of the constitutively-active AtCPK3CA-RFP had a stronger effect on PVXGFP spreading and to a similar degree with the over-expression of REM1.3 alone (Fig 6D). AtCPK3's function towards PVX movement was observed to be clearly mediated by its kinase activity, as the expression of the catalytically inactive mutant AtCPK3CA ${ }^{\mathrm{D} 202 \mathrm{~A}}$ had no effect on PVX-GFP propagation (Fig 6D).

This raised the question whether the effect of AtCPK3CA on PVX propagation was REM-dependent. To tackle this question, we stably transformed $N$. benthamiana plants with a hairpin construct, to induce post-transcriptional gene silencing, which resulted in lowering RNA and protein expression of group 1 endogenous NbREMs (Fig EV7A,B). Consistent with previous studies ${ }^{7}$, silencing of group 1 REM correlates with an increase of PVX cell-to-cell movement (Fig EV7C). Importantly, PVX assays demonstrated that AtCPK3CA ability to restrict PVX movement was impaired in two independent $N$. benthamiana lines underexpressing group 1 REM levels, namely lines 1.4 and 10.2 (with expression levels decreased respectively by 2 and 20 times) (Fig 6E), indicating that REMs might be the direct substrate of CPK3 in vivo. Altogether, these data suggest that CPK3 and group 1 REMs are major regulators involved in signaling and antiviral defense at the PM level. 


\section{Discussion}

Protein phosphorylation is a ubiquitous and specific mechanism of cell communication ${ }^{67}$. The addition of a phosphate group on one or more critical residues of a given protein can induce important conformational changes that affect energetically favorable interactions and may lead to changes in its interacting network, localization, abundance and may influence the activity of protein signaling pools 68. Although, since the initial discovery of REM1.3 in 1989, accumulating evidence suggests that the functions of REM proteins are regulated by protein phosphorylation ${ }^{33-35}$, the biological significance of this phosphorylation remained unclear to this date. REM proteins were among the first plant proteins described which supported the notion of PM subcompartmentalization to functional proteinlipid nanodomains ${ }^{7,1069}$, also named membrane rafts ${ }^{2,3}$. In the present paper, we used REM1.3 and PVX as an experimental system to study the role of protein phosphorylation and membrane dynamics in the context of stress response.

\section{REM functions likely involve distinct PM compartments during plant PVX-sensing}

Understanding how plants defend themselves against viruses remains a challenging field. The canonical plant immune response against viruses is mainly represented by the mechanism of RNA silencing ${ }^{70}$, , while additional mechanisms of plant antiviral defense involve hormonal signaling, protein degradation, suppression of protein synthesis and metabolic regulation ${ }^{42} 70,72$. Antiviral defense presents similarities to the immune response against microbes ${ }^{73} 7475$. Compelling evidence suggests that cell-surface as well as intracellular plant immune receptors recognize viral elicitors ${ }^{46} 76$ 7778798081 . An additional number of host cell components have been shown genetically to affect viral replication or cell-to-cell movement ${ }^{7}{ }^{82}$, indicating that more sophisticated plant defense mechanisms against viruses may exist.

For instance, manipulation of REM levels in transgenic Solanaceae, suggested that REM is as a positive regulator of defense against the PVX ${ }^{7,13,31}$ by affecting viral cell-to-cell movement. We recently showed that REM1.3 does not interfere with the suppressor ability of PVX movement protein TGBp1, but specifically affects its gating ability ${ }^{30}$. In this paper, we provide supporting mechanistic evidence that REM1.3 regulates the levels of callose accumulation at PD pit fields during PVX infection (Fig 1). Whether this function is mediated by a direct interaction with callose synthase/glucanase complexes remains however still unknown. Surprisingly, we found that REM1.3 is not dramatically recruited to PD pit fields, although its PD index is slightly increased after PVX infection (Fig 1). The spt-PALM VAEM microscopy data supports an increase of protein mobility and redistribution to distinct domains during PVX infection (Fig 1). These findings indicate the existence of a mechanism that operates at specific REM1.3-associated PM nanodomains, capable of regulating PD permeability (Fig 1). The dynamic partitioning between PM nanodomains and PD pit fields needs to be further studied. 


\section{Plant PVX-sensing induces the activation of a calcium-dependent protein kinase}

Since various studies have reported REM phosphorylation during plant-microbe interactions 14153435 , we set out to address which kinase phosphorylates REM and whether REM1.3 phosphorylation plays a role in REM-mediated anti-viral defense. Indeed, our experimental findings show that plant PVX sensing induces the activation of a membrane-bound calcium-dependent protein kinase that in turn phosphorylates REM1.3 (Fig 2, Fig EV2, Fig 5). Importantly, we show that the kinase able to phosphorylate REM 1.3 is activated specifically by the expression of two PVX proteins, namely CP and TGBp1. Deciphering the exact mechanisms allowing the molecular recognition of those PVX components will be a crucial step toward understanding REM-mediated anti-viral defense.

Genetic studies have established that different CPKs comprise critical plant signaling hubs by sensing and translating pathogen-induced changes of calcium concentrations $59{ }^{60}$. Biochemical characterization of the kinase phosphorylating 6His-REM1.3 showed that its strong sensitivity to calcium (Figure 5C) corresponds to homologs of phylogenetic subgroup II CPKs from Arabidopsis ${ }^{59}$. CPK3 is a prominent member of subgroup II, shown to function in stomatal ABA signaling ${ }^{83}$, in salt stress response ${ }^{63} 84$ and in a defense response against an herbivore ${ }^{85}$. Interestingly, it was suggested that AtREM1.3 from taxonomical group 1 of REMs could be a substrate for AtCPK3 in response to salt stress ${ }^{63}$. Here we show that AtCPK3 can interact in vivo with REM1.3 (Fig 6C) and that AtCPK3 phosphorylates REM1.3 in an in vitro kinase assay (Fig 5D). Transient overexpression of AtCPK3 in $N$. benthamiana resulted in a reduction of PVX propagation in a REM-dependent manner, providing compelling evidence that CPK3 together with REM contribute to the plant antiviral immunity. This is the first report demonstrating the participation of CPKs in plant basal immunity against viruses. The activation of CPKs by PVX supports the notion that PVX similarly to other pathogens or stress factors induces changes in calcium concentrations in the cell, which are sensed by the CPKs and translated via the phosphorylation of REM and other unknown downstream components. In Nicotiana tabaccum calmodulin isoforms are critical for the plant resistance against Tobacco Mosaic Virus and Cucumber Mosaic Virus, further illustrating the existence of virus-specific patterns of calcium signals ${ }^{86}{ }^{87}$. More work is needed to identify the CPK family members participating to the response and also the nature and specificity of those PVX-induced calcium changes.

\section{Phosphorylation regulates group 1 REM's function during PVX cell-to-cell movement}

AtCPK3 specifically phosphorylated REM1.3 at its N-terminal domain (residues 1-116), a domain displaying a mostly intrinsically disordered secondary structure (Fig 3A, 5). In silico analysis followed by a mutagenesis approach coupled with in vitro kinase assays revealed three major putative phosphorylation sites for REM1.3, namely S74, T86 and S91 on REM1.3. The in vitro phosphorylation of REM1.3 (Fig 3A, 5E) is almost totally lost when S74, T86 and S91 are mutated to non-phosphorylable residues, confirming these residues as major REM1.3 phosphorylation sites. The triple phospho-null mutant, YFP-REM1.3 ${ }^{\mathrm{AAA}}$, totally obliterated REM1.3's capability to restrict PVX 
cell-to-cell movement. Reciprocally, REM1.3 triple phosphomimetic mutant YFP-REM1.3 ${ }^{\text {DDD }}$ appeared fully functional (Fig 3D, 3E). These results strongly support the functional involvement of single or combined phosphorylation in the N-terminal domain of S74, T86 and S91 to establish REM's function in the context of PVX infection. This is in contrast with LjSYMREM1 from Lotus japonicus which was shown to be phosphorylated at its C-terminal domain in vitro by SYMRK ${ }^{14}$. Despite the fact that phosphorylation of REM proteins has been widely reported 1415343548 , this work firstly describes an associated role of REM-induced phosphorylation with its function.

\section{Toward the understanding of REMORIN function.}

Our finding that overexpression of AtREM1.2 and AtREM1.3 also restricts PVX-GFP cell-to-cell movement (Fig EV5B), suggests that REM phosphorylation and its associated functions might be conserved for some REMs of taxonomic group 1b. In good agreement, AtREM1.2 and AtREM1.3 localize to the same PM nanodomains ${ }^{64}$ and maintain conserved phosphorylation sites with REM1.3 (Fig EV5A). By contrast, AtREM4.1 from subgroup 4 has an opposite effect against geminiviral propagation, promoting susceptibility to Beet Curly Top Virus and Beet Severe Curly Top Virus ${ }^{15}$, but also does not present the same in silico phosphorylation profile ${ }^{48}$.

Overexpression of REM1.3 restricts TMV propagation (Fig EV3A), and additionally hampers the gating activity of movement proteins from different virus genera ${ }^{30}$, meaning that group 1 REM proteins might act as negative regulators for more viruses. These findings suggest that the initial hypothesis that REM1.3 causes the sequestration of the PVX virions at the PD ${ }^{7}$ might not hold true, but rather that REM1.3 might have a more general role in plant stress and PD regulation (Fig 1,3). Interestingly, REM1.3 promotes susceptibility to Phytophthora infestans in $N$. benthamiana ${ }^{37}$. The exact role of REM1.3 as a common regulator of different signaling pathways and its direct role in PD remain to be determined.

It has been speculated that phosphorylation in intrinsically disorder regions of proteins may act as a molecular switch and confer potential protein-protein interaction plasticity ${ }^{68,88}$. The intrinsically disordered REM1.3 N-terminal domain exhibits the most sequence variability in REM proteins, presumably conferring signaling specificity ${ }^{29,48}$. Phosphorylation of AtREM1.3's N-terminal domain could stabilize coiled-coiled-associated protein trimerization and protein-protein interactions ${ }^{48}$. Phosphorylated REM1.3 seems to be further targeted to PD-PM to trigger callose deposition. In good agreement, we found that the mobility in the PM of REM1.3 changed depending on its phospho-status (Fig 4). The triple phosphomimetic mutant exhibited a less confined and more mobile behavior at the PM, reminiscent of the WT protein in the context of PVX infection (Fig 4D). Similarly to the role of 14.3.3 proteins in plants ${ }^{89}$, REM1.3 could act as a scaffolding protein, interacting with multiple members of a signaling pathway and tethering them into complexes to specific areas of the membrane. Hence, REM1.3 phosphorylation could act as a regulatory switch of protein conformations that would modulate REM1.3 specific interaction patterns and transient signalosomes at the PM. The 

available under aCC-BY-NC-ND 4.0 International license.

triple phosphomimetic REM mutant might reflect a 'functionally active' form that constitutes REMguided signalosomes against PVX-infection at the PM and should be exploited in future studies. The study of the phosphorylation-dependent interactions of REM1.3 (and related phosphocode) in regards to the modulation of REM1.3 PM dynamics and molecular function is the topic for future studies. 


\section{Author contributions}

AP performed the phosphorylation experiments. JG performed the spt-PALM experiments, PD index and callose measurements. JG and AP produced and imaged the REM mutants and built the figures. PG performed the BiFC assays. MB carried out the phosphorylation experiments with CPK3. AP, JG, AFD, VS performed the experiments of virus propagation. PG, AFD and VG produced the $N$. benthamiana hpREM-lines. MB, SGR, CZ, EEB and VG provided scientific expertise. AP, JG, SM and VG wrote the paper with the help of all the authors. VG and SM supervised the research. All authors read, edited, and approved the manuscript.

\section{Conflict of interest}

No conflict of interest declared.

\section{Acknowledgments}

Imaging was performed at the Bordeaux Imaging Center, member of the national infrastructure France BioImaging. AP was supported by the Greek fellowship program IKY in France. JG, PG and AFD are/were supported by the Ministère de l'Enseignement Supérieur et de la Recherche, France (MERS, doctoral grants). We acknowledge Dinesh Kumar for the gift of TMV-GFP clone , Alicia Zelada for the gift of PVX $\triangle \mathrm{TGBp} 1$ and viral protein constructs and Coralie Chesseron for greenhouse facilities. The IPS2 is supported by the LabEx Saclay Plant Sciences-SPS (ANR-10-LABX-0040SPS). VG, SM, EEB, VS and VG are supported by the French ANR project "Potymove" (ANR-16CE20-008-01). EEB, VG and SM are supported by the French ANR project "Connect". CZ is supported by the Gatsby Charitable Foundation and the European Research Council (grant "PHOSPHinnATE"). 


\section{References}

1. Nicolson, G.L. The Fluid-Mosaic Model of Membrane Structure: still relevant to understanding the structure, function and dynamics of biological membranes after more than 40 years. Biochimica et biophysica acta 1838, 1451-1466 (2014).

2. Lingwood, D. \& Simons, K. Lipid rafts as a membrane-organizing principle. Science 327, 46-50 (2010).

3. Simons, K. \& Gerl, M.J. Revitalizing membrane rafts: new tools and insights. Nature reviews. Molecular cell biology 11, 688-699 (2010).

4. Kusumi, A. et al. Dynamic organizing principles of the plasma membrane that regulate signal transduction: commemorating the fortieth anniversary of Singer and Nicolson's fluid-mosaic model. Annu Rev Cell Dev Biol 28, 215-250 (2012).

5. Mugler, A., Tostevin, F. \& ten Wolde, P.R. Spatial partitioning improves the reliability of biochemical signaling. Proceedings of the National Academy of Sciences of the United States of America 110, 5927-5932 (2013).

6. Bucherl, C.A. et al. Plant immune and growth receptors share common signalling components but localise to distinct plasma membrane nanodomains. Elife 6 (2017).

7. Raffaele, S. et al. Remorin, a solanaceae protein resident in membrane rafts and plasmodesmata, impairs potato virus X movement. The Plant cell 21, 1541-1555 (2009).

8. Jarsch, I.K. \& Ott, T. Perspectives on remorin proteins, membrane rafts, and their role during plant-microbe interactions. Molecular plant-microbe interactions : MPMI 24, 7-12 (2011).

9. Jarsch, I.K. et al. Plasma Membranes Are Subcompartmentalized into a Plethora of Coexisting and Diverse Microdomains in Arabidopsis and Nicotiana benthamiana. The Plant cell 26, 1698-1711 (2014).

10. Lefebvre, B. et al. A remorin protein interacts with symbiotic receptors and regulates bacterial infection. Proceedings of the National Academy of Sciences of the United States of America 107, 2343-2348 (2010).

11. Gui, J. et al. OsREM4.1 Interacts with OsSERK1 to Coordinate the Interlinking between Abscisic Acid and Brassinosteroid Signaling in Rice. Dev Cell 38, 201-213 (2016).

12. Raffaele, S., Perraki, A. \& Mongrand, S. The Remorin C-terminal Anchor was shaped by convergent evolution among membrane binding domains. Plant signaling \& behavior 8 (2013).

13. Perraki, A. et al. Plasma membrane localization of Solanum tuberosum remorin from group 1 , homolog 3 is mediated by conformational changes in a novel C-terminal anchor and required for the restriction of potato virus $\mathrm{X}$ movement]. Plant physiology 160, 624-637 (2012).

14. Toth, K. et al. Functional domain analysis of the Remorin protein LjSYMREM1 in Lotus japonicus. PloS one 7, e30817 (2012).

15. Son, S., Oh, C.J. \& An, C.S. Arabidopsis thaliana Remorins Interact with SnRK1 and Play a Role in Susceptibility to Beet Curly Top Virus and Beet Severe Curly Top Virus. The plant pathology journal 30, 269-278 (2014).

16. Jamann, T.M. et al. A remorin gene is implicated in quantitative disease resistance in maize. Theor Appl Genet 129, 591-602 (2016).

17. Checker, V.G. \& Khurana, P. Molecular and functional characterization of mulberry EST encoding remorin (MiREM) involved in abiotic stress. Plant Cell Rep 32, 17291741 (2013). 
18. Yue, J., Li, C., Liu, Y.\& Yu, J. A remorin gene SiREM6, the target gene of SiARDP, from foxtail millet (Setaria italica) promotes high salt tolerance in transgenic Arabidopsis. PloS one 9, e100772 (2014).

19. Fernandez-Calvino, L. et al. Arabidopsis plasmodesmal proteome. PloS one 6, e18880 (2011).

20. Gui, J., Zheng, S., Shen, J. \& Li, L. Grain setting defect1 (GSD1) function in rice depends on S-acylation and interacts with actin 1 (OsACT1) at its C-terminal. Frontiers in plant science 6, 804 (2015).

21. Tilsner, J., Nicolas, W., Rosado, A. \& Bayer, E.M. Staying Tight: Plasmodesmal Membrane Contact Sites and the Control of Cell-to-Cell Connectivity in Plants. Annu Rev Plant Biol 67, 337-364 (2016).

22. Nicolas, W.J. et al. Architecture and permeability of post-cytokinesis plasmodesmata lacking cytoplasmic sleeves. Nat Plants 3, 17082 (2017).

23. Grison, M.S. et al. Specific membrane lipid composition is important for plasmodesmata function in Arabidopsis. The Plant cell 27, 1228-1250 (2015).

24. Lee, J.Y. et al. A plasmodesmata-localized protein mediates crosstalk between cell-tocell communication and innate immunity in Arabidopsis. The Plant cell 23, 33533373 (2011).

25. Zavaliev, R., Dong, X. \& Epel, B.L. Glycosylphosphatidylinositol (GPI) Modification Serves as a Primary Plasmodesmal Sorting Signal. Plant physiology 172, 1061-1073 (2016).

26. Chen, X.Y. \& Kim, J.Y. Callose synthesis in higher plants. Plant signaling \& behavior 4, 489-492 (2009).

27. Angell, S.M., Davies, C. \& Baulcombe, D.C. Cell-to-cell movement of potato virus X is associated with a change in the size-exclusion limit of plasmodesmata in trichome cells of Nicotiana clevelandii. Virology 216, 197-201 (1996).

28. Howard, A.R. et al. Potato virus X TGBp1 induces plasmodesmata gating and moves between cells in several host species whereas CP moves only in N. benthamiana leaves. Virology 328, 185-197 (2004).

29. Raffaele, S., Mongrand, S., Gamas, P., Niebel, A. \& Ott, T. Genome-wide annotation of remorins, a plant-specific protein family: evolutionary and functional perspectives. Plant physiology 145, 593-600 (2007).

30. Perraki, A. et al. StRemorin1.3 hampers Potato virus X TGBp1 ability to increase plasmodesmata permeability, but does not interfere with its silencing suppressor activity. FEBS letters 588, 1699-1705 (2014).

31. Gronnier, J. et al. Structural basis for plant plasma membrane protein dynamics and organization into functional nanodomains. Elife 6 (2017).

32. Farmer, E.E., Moloshok, T.D., Saxton, M.J. \& Ryan, C.A. Oligosaccharide signaling in plants. Specificity of oligouronide-enhanced plasma membrane protein phosphorylation. The Journal of biological chemistry 266, 3140-3145 (1991).

33. Reymond, P. et al. Cloning of a cDNA encoding a plasma membrane-associated, uronide binding phosphoprotein with physical properties similar to viral movement proteins. The Plant cell 8, 2265-2276 (1996).

34. Benschop, J.J. et al. Quantitative phosphoproteomics of early elicitor signaling in Arabidopsis. Molecular \& cellular proteomics : MCP 6, 1198-1214 (2007).

35. Widjaja, I. et al. Combining subproteome enrichment and Rubisco depletion enables identification of low abundance proteins differentially regulated during plant defense. Proteomics 9, 138-147 (2009).

36. Tilsner, J. et al. Replication and trafficking of a plant virus are coupled at the entrances of plasmodesmata. J Cell Biol 201, 981-995 (2013). 
37. Bozkurt, T.O. et al. The Plant Membrane-Associated REMORIN1.3 Accumulates in Discrete Perihaustorial Domains and Enhances Susceptibility to Phytophthora infestans. Plant physiology 165, 1005-1018 (2014).

38. Kusumi, A. \& Suzuki, K. Toward understanding the dynamics of membrane-raftbased molecular interactions. Biochimica et biophysica acta 1746, 234-251 (2005).

39. Martiniere, A. et al. Cell wall constrains lateral diffusion of plant plasma-membrane proteins. Proceedings of the National Academy of Sciences of the United States of America 109, 12805-12810 (2012).

40. Konrad, S.S. et al. S-acylation anchors remorin proteins to the plasma membrane but does not primarily determine their localization in membrane microdomains. The New phytologist 203, 758-769 (2014).

41. Levet, F. et al. SR-Tesseler: a method to segment and quantify localization-based super-resolution microscopy data. Nat Methods 12, 1065-1071 (2015).

42. Calil, I.P. \& Fontes, E.P.B. Plant immunity against viruses: antiviral immune receptors in focus. Ann Bot 119, 711-723 (2017).

43. Nicaise, V. \& Candresse, T. Plum pox virus capsid protein suppresses plant pathogenassociated molecular pattern (PAMP)-triggered immunity. Molecular plant pathology 18, 878-886 (2017).

44. de Ronde, D., Butterbach, P. \& Kormelink, R. Dominant resistance against plant viruses. Frontiers in plant science 5, 307 (2014).

45. de Ronde, D. et al. Analysis of Tomato spotted wilt virus NSs protein indicates the importance of the N-terminal domain for avirulence and RNA silencing suppression. Molecular plant pathology 15, 185-195 (2014).

46. Gouveia, B.C., Calil, I.P., Machado, J.P., Santos, A.A. \& Fontes, E.P. Immune Receptors and Co-receptors in Antiviral Innate Immunity in Plants. Front Microbiol 7, 2139 (2016).

47. Niehl, A. et al. Fluorescent Tobacco mosaic virus-Derived Bio-Nanoparticles for Intravital Two-Photon Imaging. Frontiers in plant science 6, 1244 (2015).

48. Marin, M. \& Ott, T. Phosphorylation of intrinsically disordered regions in remorin proteins. Frontiers in plant science 3, 86 (2012).

49. Cluzet, S. Etude du rôle de la rémorine dans la défense végétale. Thèse de doctorat, Université de Lausanne (2001).

50. Mongrand, S. et al. Lipid rafts in higher plant cells: purification and characterization of Triton X-100-insoluble microdomains from tobacco plasma membrane. The Journal of biological chemistry 279, 36277-36286 (2004).

51. Kalay, Z. Reaction kinetics in the plasma membrane. Biotechnol J 7, 745-752 (2012).

52. Tian, T. et al. Plasma membrane nanoswitches generate high-fidelity Ras signal transduction. Nature cell biology 9, 905-914 (2007).

53. Furt, F. et al. Polyphosphoinositides are enriched in plant membrane rafts and form microdomains in the plasma membrane. Plant physiology 152, 2173-2187 (2010).

54. Meggio, F. et al. Different susceptibility of protein kinases to staurosporine inhibition. Kinetic studies and molecular bases for the resistance of protein kinase CK2. European journal of biochemistry / FEBS 234, 317-322 (1995).

55. Ruegg, U.T. \& Burgess, G.M. Staurosporine, K-252 and UCN-01: potent but nonspecific inhibitors of protein kinases. Trends Pharmacol Sci 10, 218-220 (1989).

56. Meggio, F. \& Pinna, L.A. One-thousand-and-one substrates of protein kinase CK2? FASEB journal : official publication of the Federation of American Societies for Experimental Biology 17, 349-368 (2003). 
57. Binder, B.M., Harper, J.F. \& Sussman, M.R. Characterization of an Arabidopsis calmodulin-like domain protein kinase purified from Escherichia coli using an affinity sandwich technique. Biochemistry 33, 2033-2041 (1994).

58. Ren, Z. et al. Direct determination of phosphatase activity from physiological substrates in cells. PloS one 10, e0120087 (2015).

59. Boudsocq, M. \& Sheen, J. CDPKs in immune and stress signaling. Trends in plant science 18, 30-40 (2013).

60. Schulz, P., Herde, M. \& Romeis, T. Calcium-dependent protein kinases: hubs in plant stress signaling and development. Plant physiology 163, 523-530 (2013).

61. Boudsocq, M., Droillard, M.J., Regad, L. \& Lauriere, C. Characterization of Arabidopsis calcium-dependent protein kinases: activated or not by calcium? The Biochemical journal 447, 291-299 (2012).

62. Kierszniowska, S., Seiwert, B. \& Schulze, W.X. Definition of Arabidopsis sterol-rich membrane microdomains by differential treatment with methyl-beta-cyclodextrin and quantitative proteomics. Molecular \& cellular proteomics : MCP 8, 612-623 (2009).

63. Mehlmer, N. et al. The $\mathrm{Ca}(2+)$-dependent protein kinase $\mathrm{CPK} 3$ is required for MAPK-independent salt-stress acclimation in Arabidopsis. The Plant journal : for cell and molecular biology 63, 484-498 (2010).

64. Demir, F. et al. Arabidopsis nanodomain-delimited ABA signaling pathway regulates the anion channel SLAH3. Proceedings of the National Academy of Sciences of the United States of America 110, 8296-8301 (2013).

65. Harmon, A.C., Gribskov, M. \& Harper, J.F. CDPKs - a kinase for every Ca2+ signal? Trends in plant science 5, 154-159 (2000).

66. Harper, J.F., Breton, G. \& Harmon, A. Decoding $\mathrm{Ca}(2+)$ signals through plant protein kinases. Annu Rev Plant Biol 55, 263-288 (2004).

67. Ubersax, J.A. \& Ferrell, J.E., Jr. Mechanisms of specificity in protein phosphorylation. Nature reviews. Molecular cell biology 8, 530-541 (2007).

68. Bah, A. et al. Folding of an intrinsically disordered protein by phosphorylation as a regulatory switch. Nature 519, 106-109 (2015).

69. Gui, J., Liu, C., Shen, J. \& Li, L. Grain setting defect1, encoding a remorin protein, affects the grain setting in rice through regulating plasmodesmatal conductance. Plant physiology 166, 1463-1478 (2014).

70. Incarbone, M. \& Dunoyer, P. RNA silencing and its suppression: novel insights from in planta analyses. Trends in plant science 18, 382-392 (2013).

71. Carr, J.P. \& Loebenstein, G. Natural and engineered resistance to plant viruses, part II. Preface. Adv Virus Res 76, vii (2010).

72. Zorzatto, C. et al. NIK1-mediated translation suppression functions as a plant antiviral immunity mechanism. Nature 520, 679-682 (2015).

73. Zipfel, C. Plant pattern-recognition receptors. Trends Immunol 35, 345-351 (2014).

74. Dodds, P.N. \& Rathjen, J.P. Plant immunity: towards an integrated view of plantpathogen interactions. Nat Rev Genet 11, 539-548 (2010).

75. Dangl, J.L., Horvath, D.M. \& Staskawicz, B.J. Pivoting the plant immune system from dissection to deployment. Science 341, 746-751 (2013).

76. Kroner, A., Hamelin, G., Andrivon, D. \& Val, F. Quantitative resistance of potato to Pectobacterium atrosepticum and Phytophthora infestans: integrating PAMP-triggered response and pathogen growth. PloS one 6, e23331 (2011).

77. Hanssen, I.M. et al. Differential tomato transcriptomic responses induced by pepino mosaic virus isolates with differential aggressiveness. Plant physiology 156, 301-318 (2011). 
78. Love, A.J., Yun, B.W., Laval, V., Loake, G.J. \& Milner, J.J. Cauliflower mosaic virus, a compatible pathogen of Arabidopsis, engages three distinct defense-signaling pathways and activates rapid systemic generation of reactive oxygen species. Plant physiology 139, 935-948 (2005).

79. Whitham, S.A. et al. Diverse RNA viruses elicit the expression of common sets of genes in susceptible Arabidopsis thaliana plants. The Plant journal : for cell and molecular biology 33, 271-283 (2003).

80. Jones, J.D. \& Dangl, J.L. The plant immune system. Nature 444, 323-329 (2006).

81. Mandadi, K.K. \& Scholthof, K.B. Plant immune responses against viruses: how does a virus cause disease? The Plant cell 25, 1489-1505 (2013).

82. Levy, A., Zheng, J.Y. \& Lazarowitz, S.G. Synaptotagmin SYTA forms ER-plasma membrane junctions that are recruited to plasmodesmata for plant virus movement. Current biology : CB 25, 2018-2025 (2015).

83. Mori, I.C. et al. CDPKs CPK6 and CPK3 function in ABA regulation of guard cell Stype anion- and $\mathrm{Ca}(2+)$-permeable channels and stomatal closure. PLoS Biol 4, e327 (2006).

84. Latz, A. et al. Salt stress triggers phosphorylation of the Arabidopsis vacuolar K+ channel TPK1 by calcium-dependent protein kinases (CDPKs). Molecular plant 6, 1274-1289 (2013).

85. Kanchiswamy, C.N. et al. Regulation of Arabidopsis defense responses against Spodoptera littoralis by CPK-mediated calcium signaling. BMC plant biology 10, 97 (2010).

86. Takabatake, R. et al. Pathogen-induced calmodulin isoforms in basal resistance against bacterial and fungal pathogens in tobacco. Plant Cell Physiol 48, 414-423 (2007).

87. Nakahara, K.S. et al. Tobacco calmodulin-like protein provides secondary defense by binding to and directing degradation of virus RNA silencing suppressors. Proceedings of the National Academy of Sciences of the United States of America 109, 1011310118 (2012).

88. Iakoucheva, L.M. et al. The importance of intrinsic disorder for protein phosphorylation. Nucleic acids research 32, 1037-1049 (2004).

89. Cotelle, V. \& Leonhardt, N. 14-3-3 Proteins in Guard Cell Signaling. Frontiers in plant science 6, 1210 (2015).

90. Scholthof, K.B. et al. Top 10 plant viruses in molecular plant pathology. Molecular plant pathology 12, 938-954 (2011).

91. Hosy, E., Martiniere, A., Choquet, D., Maurel, C. \& Luu, D.T. Super-resolved and dynamic imaging of membrane proteins in plant cells reveal contrasting kinetic profiles and multiple confinement mechanisms. Molecular plant 8, 339-342 (2015).

92. Claro, E., Sarri, E. \& Picatoste, F. Measurement of phospholipase C activity in brain membranes. Methods in molecular biology 41, 177-188 (1995).

93. Boudsocq, M. et al. Differential innate immune signalling via $\mathrm{Ca}(2+)$ sensor protein kinases. Nature 464, 418-422 (2010).

94. Taton, M., Husselstein, T., Benveniste, P. \& Rahier, A. Role of highly conserved residues in the reaction catalyzed by recombinant Delta7-sterol-C5(6)-desaturase studied by site-directed mutagenesis. Biochemistry 39, 701-711 (2000).

95. Martin, K. et al. Transient expression in Nicotiana benthamiana fluorescent marker lines provides enhanced definition of protein localization, movement and interactions in planta. The Plant journal : for cell and molecular biology 59, 150-162 (2009). 
96. Peart, J.R. et al. Ubiquitin ligase-associated protein SGT1 is required for host and nonhost disease resistance in plants. Proceedings of the National Academy of Sciences of the United States of America 99, 10865-10869 (2002).

97. Sparkes, I.A., Runions, J., Kearns, A. \& Hawes, C. Rapid, transient expression of fluorescent fusion proteins in tobacco plants and generation of stably transformed plants. Nat Protoc 1, 2019-2025 (2006). 

available under aCC-BY-NC-ND 4.0 International license.

A
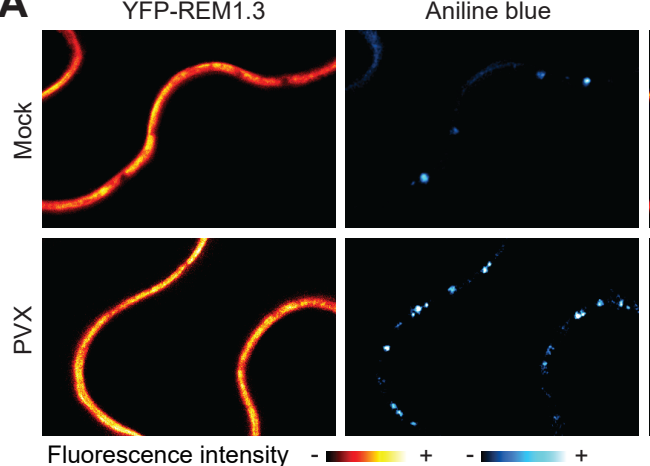

C

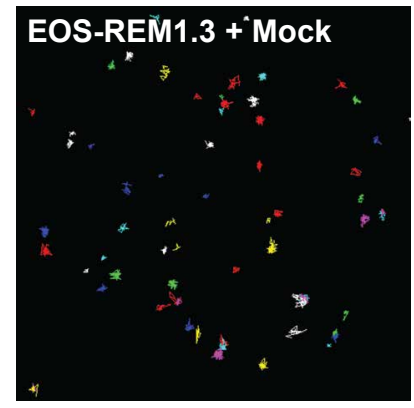

$\mathbf{F}$

G
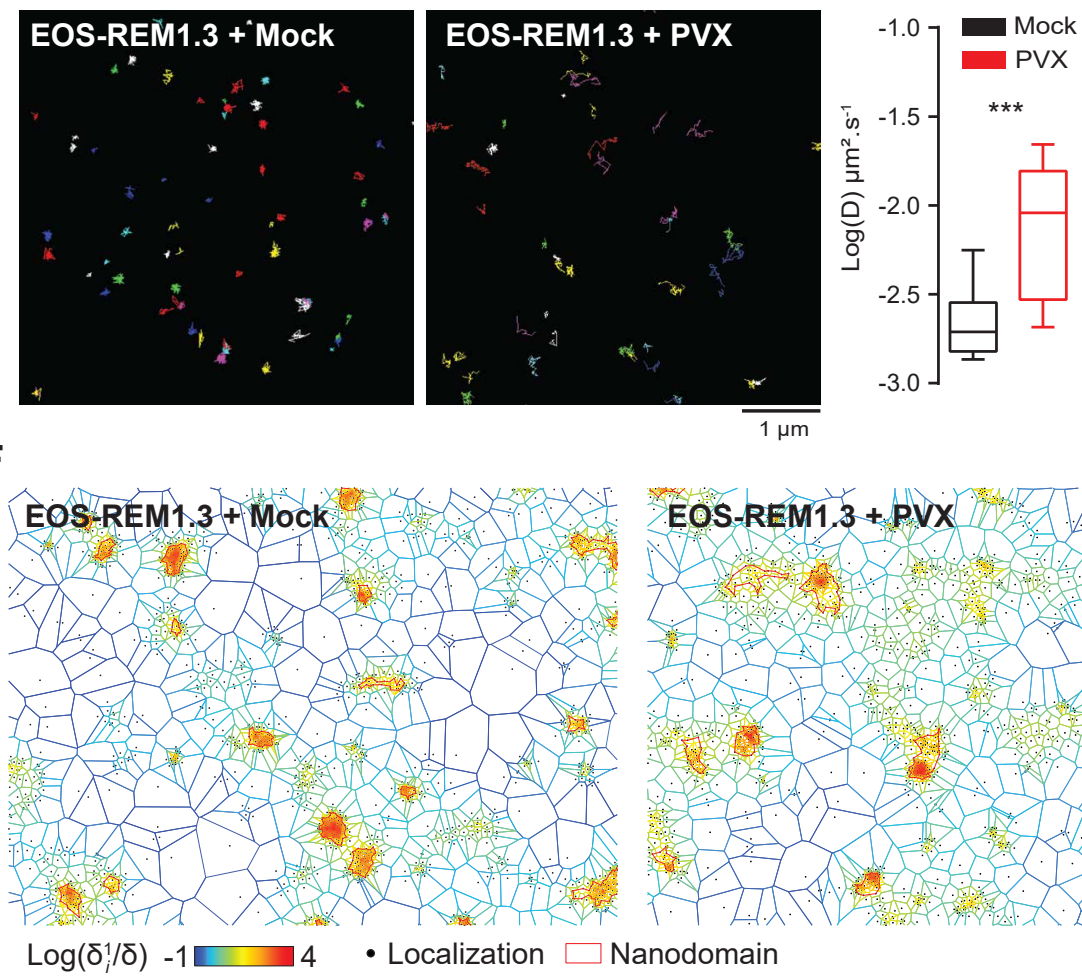

B

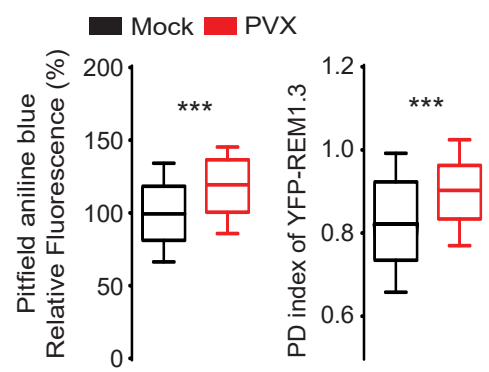

E

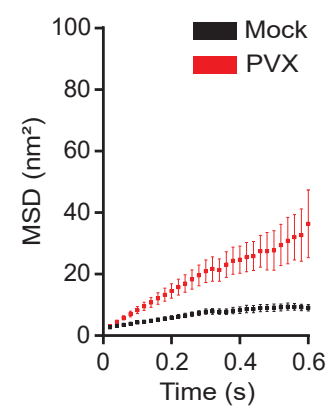

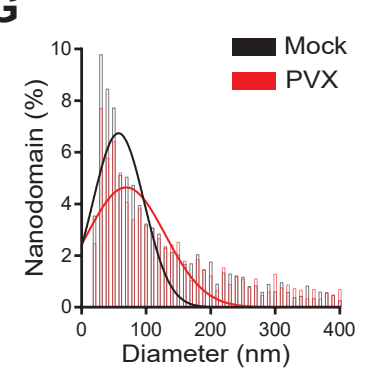
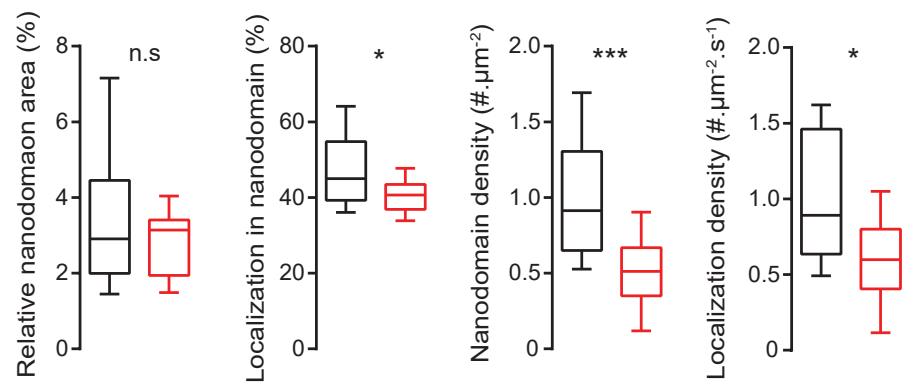

Figure 1: REM1.3 modulates Plasmodesmata callose accumulation and displays altered PM organization and dynamic following PVX infection 
Figure 1: REM1.3 modulates Plasmodesmata callose accumulation and displays altered PM organization and dynamic following PVX infection

(A) Representative confocal images of aniline blue stained $N$. benthamiana leaf epidermal cells stably expressing YFP-REM1.3 in the absence (mock is infiltration with empty A. tumefaciens) or the presence of PVX at 2 days after infiltration (DAI). Color-coding indicates fluorescence intensity.

(B) left, Pit field aniline blue fluorescence intensity was quantified by ImageJ as described in Fig EV1 and expressed as the percentage of the mock control. right, Quantification of the PD residency of YFP-REM1.3 in the absence (mock) and in the presence of PVX using the PD index ${ }^{25}$ as described in Fig EV1. Graphs represent quantifications from 3 independent biological experiments. At least 15 cells per condition were analyzed per experiment. Significant differences were determined by MannWhitney comparisons test $* * * \mathrm{p}<0.001$.

(C) Super-resolved trajectories of EOS-REM1.3 molecules in the PM plane in the absence (Mock) and presence of PVX obtained by high-resolution microscopy spt-PALM.

(D) Diffusion coefficients (D) of EOS-REM1.3 expressed as $\log (\mathrm{D})$ in the absence (Mock) and presence of PVX. Statistical significances were assessed by Mann-Whitney test $* * * p<0.001$ using data collected over two independents experiments.

(E) Mean Square Displacement (MSD) over time for the global trajectories of EOS-REM1.3 followed during at least $600 \mathrm{~ms}$ reflecting two independent experiments.

(F) Live PALM analysis of EOS-REM1.3 localization in the absence (mock) and presence of PVX by tessellation-based automatic segmentation of super-resolution images.

(G) Computation of EOS-REM1.3 single molecule organization features based on tessellation-based automatic segmentation images. For REM1.3 nanodomain size distribution for the indicated conditions, the Gaussian fits in absence (mock) and presence of PVX are indicated by lines. Total nanodomain area is expressed as percentage of the total PM surface. Percentage of EOS-REM1.3 molecules localizing into nanodomains, relative to all molecules observed. Localization density refers to the number of molecules observed per $\mu \mathrm{m}^{2}$ per second. Statistics were performed on at least 10 data sets per condition, from two independent experiments. Significant differences were determined by Mann-Whitney test $* \mathrm{p}<0.05, * * * \mathrm{p}<0.001$. 
bioRxiv preprint doi: https://doi.org/10.1101/205765; this version posted October 19,2017 . The copyright holder for this preprint (which was not certified by peer review) is the author/funder, who has granted bioRxiv a license to display the preprint in perpetuity. It is made
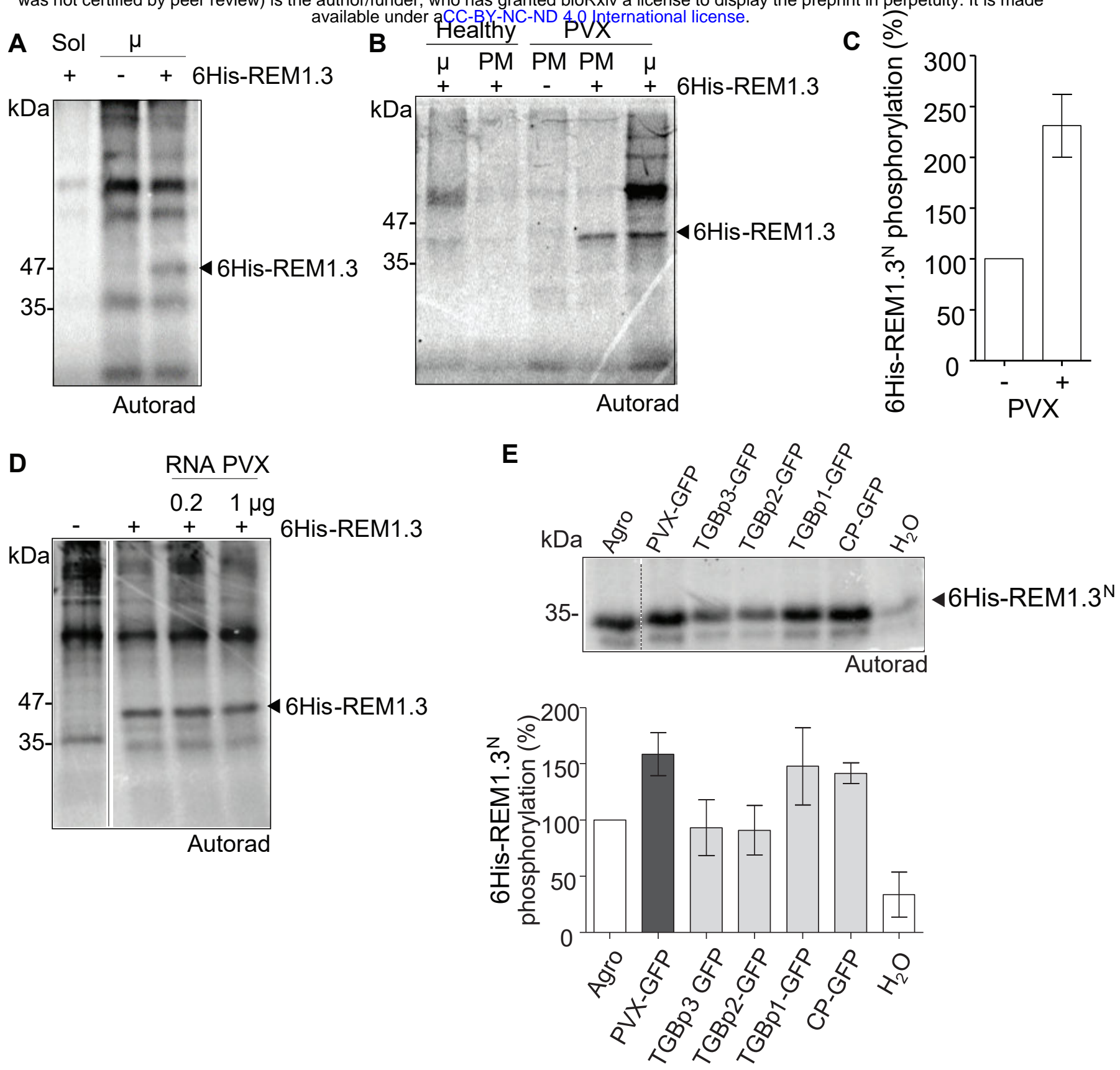

Figure 2. PVX and viral proteins induce REM1.3 phosphorylation in its N-terminal domain. 


\section{Figure 2. PVX and viral proteins induce REM1.3 phosphorylation in its N-terminal domain.}

(A, B) In vitro protein phosphorylation assays were performed by incubation of recombinant affinitypurified 6His-REM1.3 and $N$. benthamiana extracts with $\left[\gamma-{ }^{33} \mathrm{P}\right]$-ATP. The samples were run on SDSPAGE gels and developed by autoradiography. Soluble (Sol) or microsomal $(\mu)$ extracts of healthy leaves in (A), or microsomal and PM extracts from healthy and PVX-infected plants in (B) were used. (C) In vitro phosphorylation of 6 His-REM1.3 $3^{\mathrm{N}}$ by leaf microsomal extracts of healthy or PVXinfected N.benthamiana leaves. Bars show the quantification of phosphorylated 6 His-REM1. $3^{\mathrm{N}}$ bands from 5 independent repeats.

(D) In vitro phosphorylation of 6His-REM1.3 by leaf microsomal extracts in the presence of total RNA extracts from PVX-infected leaves.

(E) 6 His-REM $1.3^{\mathrm{N}}$ phosphorylation by microsomal extracts infected with PVX-GFP or expressing the indicated viral proteins at 4 DAI. Leaves expressing GFP alone, infiltrated with water or with $A$. tumefaciens strain GV3101 alone served as controls. Graph represents the quantification of 6HisREM $1.3^{\mathrm{N}}$ bands from 3 independent repeats, as a percentage of the activity induced by PVX-GFP.

In all experiments $10 \mu \mathrm{g}$ of total protein extracts and $1 \mu \mathrm{g}$ of affinity purified 6His-REM1.3 or 6HisREM $1.3^{\mathrm{N}}$ were loaded per lane. 


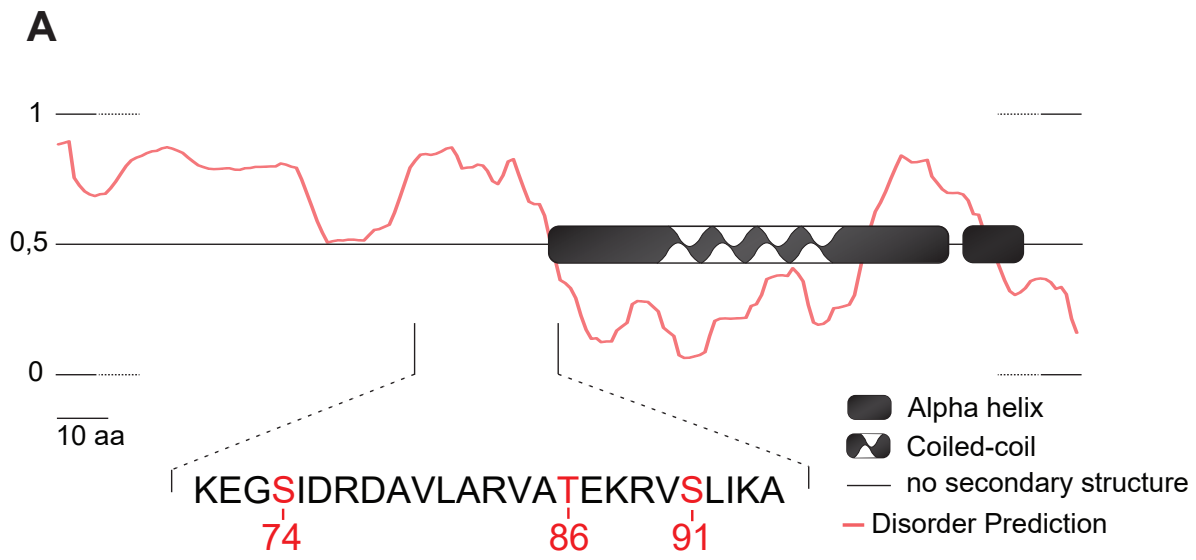

B

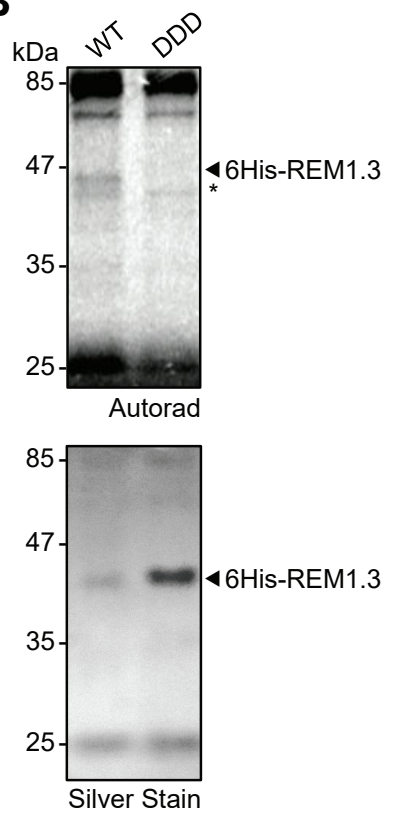

C

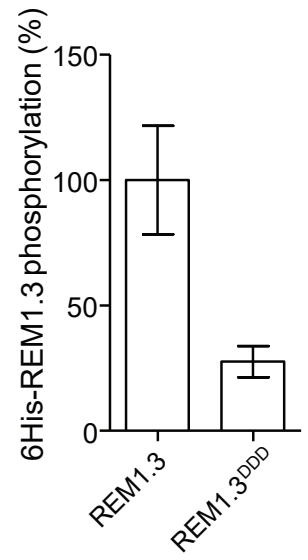

D

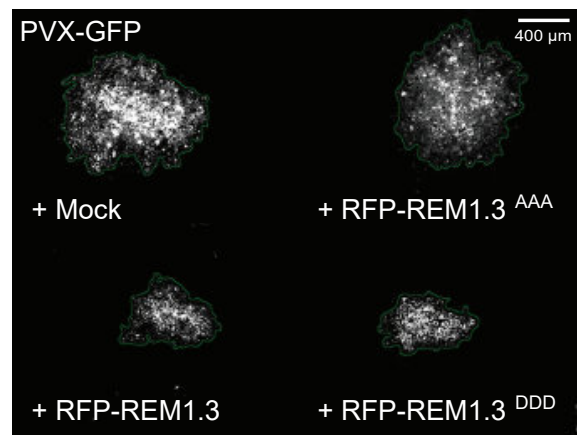

$E$

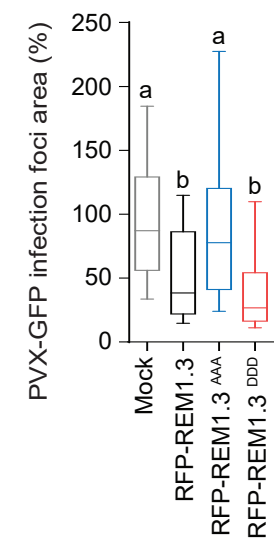

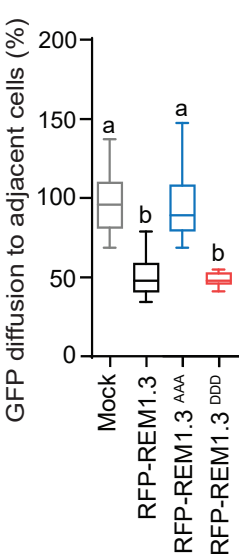

Figure 3. Mutational analysis reveals three critical phospho-residues required for REM1.3 regulation of PVX-GFP propagation and PD conductance. 
Figure 3. Mutational analysis reveals three critical phospho-residues required for REM1.3 regulation of PVX-GFP propagation and PD conductance.

(A) In silico analysis of REM1.3 protein sequence. Prediction of putative phosphorylation sites was performed by Diphos, DEPP and NETPHOS coupled with published MS data. Predictions highlight three residues S74, T86 and S91 with high probability to be phosphorylated. Disordered prediction was performed by pDONR VL XT. Numbers indicate amino acid position. (B) In vitro kinase assay on recombinant affinity purified 6 His-REM1.3 or 6 His-REM1.3 $3^{\text {DDD }}$ by incubation with $\left[\gamma^{33} \mathrm{P}\right]$-ATP and microsomal extracts of PVX-infected N. benthamiana leaves, as described in Figure 2. In (C), graph represents the relative quantifications from 4 independent reactions, using WT signal as a reference. Asterisk * indicates phosphorylation of a N. benthamiana protein of close molecular weight not detected by silver staining.

(D) Representative epifluorescence microscopy images of PVX-GFP infection foci on $N$. benthamiana leaf epidermal cells at 5 DAI. Graph represents the mean relative foci area of REM1.3 or phosphovariants compared to mock control (co-infiltration of PVX-GFP with an empty $A$. tumefaciens strain). Approximately 250 foci per condition from 5 independent biological repeats were measured Letters indicate significant differences revealed by Dunn's multiple comparisons test $\mathrm{p}<0.001$.

(E) GFP diffusion to neighbor cells was estimated by epifluorescence microscopy at 5 DAI in $N$. benthamiana cells expressing REM1.3 or phosphomutants. Measurements from 3 independent biological repeats were normalized to mock control (co-infiltration with an empty A. tumefaciens strain). Letters indicate significant differences determined by Dunn's multiple comparisons test $\mathrm{p}<0.001$. 
A

bioRxiv preprint doi: https://doi org/10.1101/205765. this version posted October 19, 2017. The copyright holder for this preprint (which was not certified by peer review) is the author/funder, who has granted bioRxiv a license to display the preprint in perpetuity. It is made
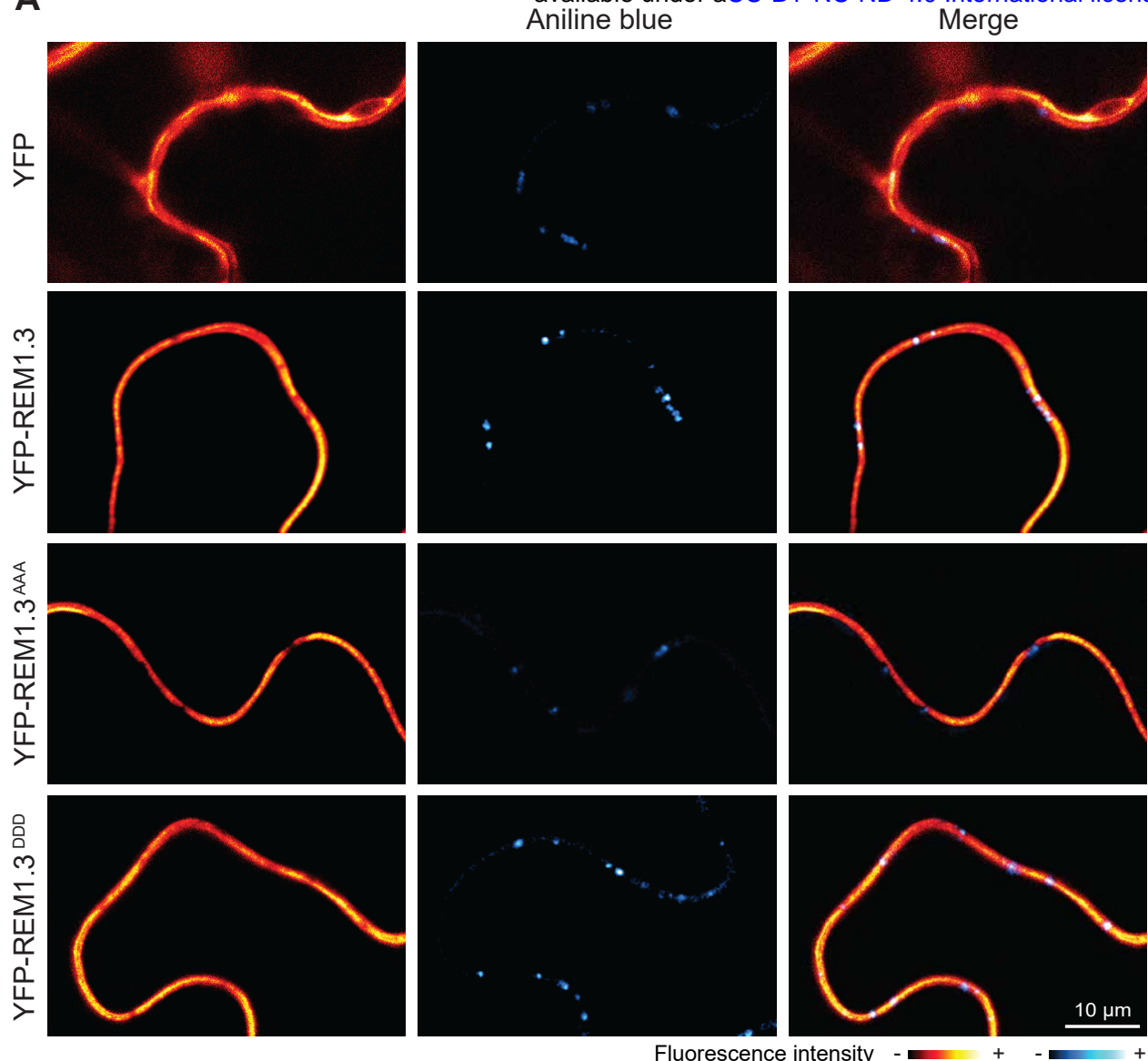

D
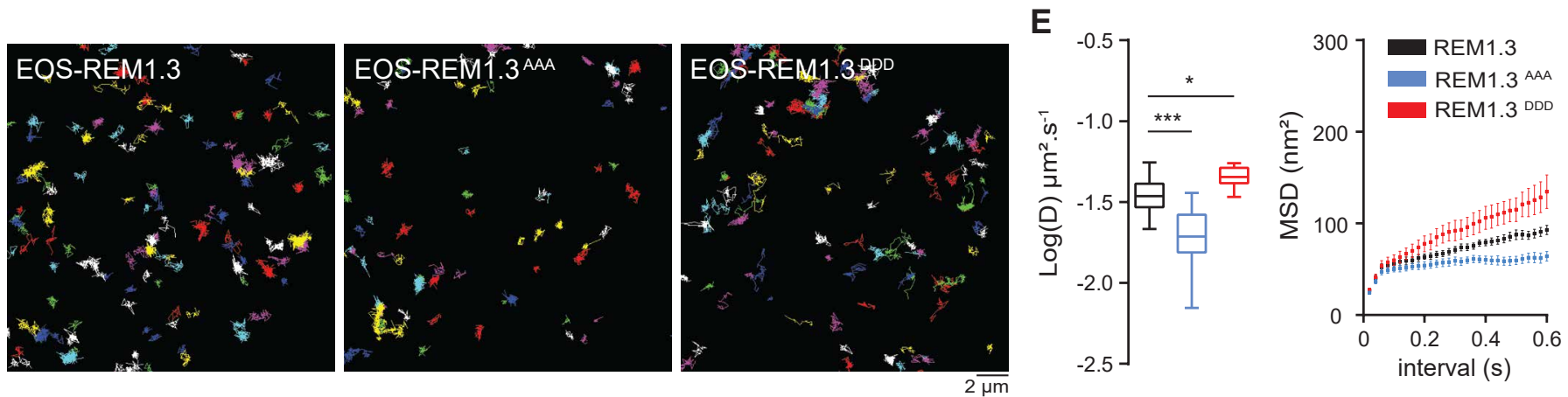

$\mathbf{F}$

EOS-REM1.3

EOS-REM1.3AAA

EOS-REM1.3 DDD

$\log \left(\delta_{i}^{1} / \delta\right)-1 \square 4 \quad \cdot$ Localization $\square$ Nanodomain

C

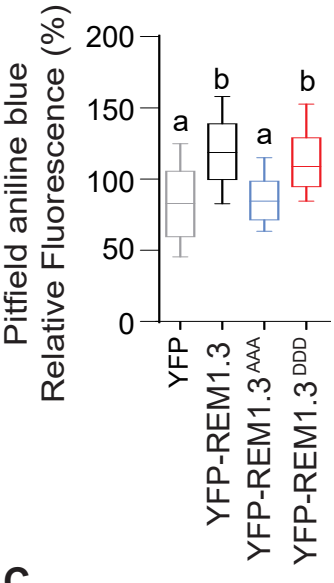

G

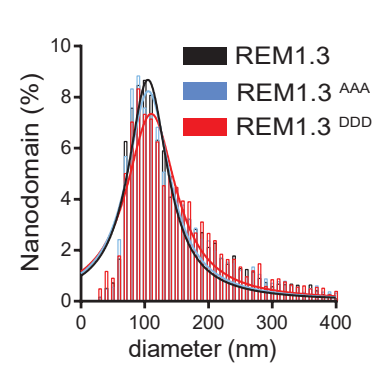

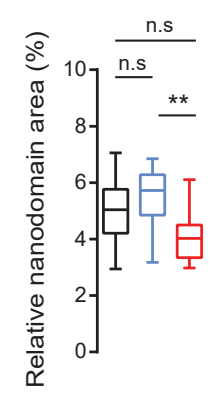

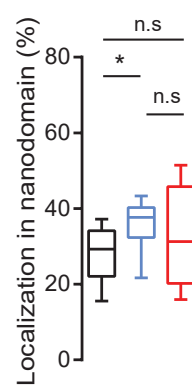




\section{Figure 4. REM1.3 dynamic localization in PD and PM nanodomains is regulated by its phospho-} status.

(A) Representative confocal mages showing aniline blue staining of callose deposition at the PD pitfields in $N$. benthamiana leaf epidermal cells expressing YFP-REM1.3 or phosphovariants. Colorcoding indicates fluorescence intensity.

(B) Graphs show aniline blue fluorescence intensities in cells transiently expressing YFP-REM1.3 and phosphomutants relative to control cells expressing YFP alone. Three independent biological experiments were performed and at least 15 cells per condition and per experiment were analyzed. Letter indicate significant differences revealed by Dunn's multiple comparisons test $\mathrm{p}<0.001$.

(C) PD index of YFP-REM1.3 phosphomutants was calculated as described in Figure EV1. Graphs present quantifications from 3 independent biological experiments. Letter indicate significant differences revealed by Dunn's multiple comparisons test $\mathrm{p}<0.002$.

(D) Super-resolved trajectories of EOS-REM1.3, and phosphomutants, transiently expressed in $N$. benthamiana cells, observed by spt-PALM. Scale bars, $2 \mu \mathrm{m}$.

(E) Distribution of diffusion coefficients (D) represented as $\log (\mathrm{D})$ of the different fusion proteins. Mean Square Displacement (MSD) over time for the global trajectories of each EOS-REM1.3 construct followed during at least $600 \mathrm{~ms}$. 27 cells for EOS-REM1.3, 15 cells for EOS-REM1.3 ${ }^{\text {AAA }}$ and 17 cells for EOS-REM1.3 $3^{\mathrm{DDD}}$ were analyzed in 3 independent experiments. Statistical analysis was performed by Mann-Whitney test * $\mathrm{p}<0.05 * * \mathrm{p}<0.01$.

(F) Live PALM analysis of EOS-REM1.3 phosphomutants by tessellation-based automatic segmentation of super-resolution images.

(G) Computation of EOS-REM1.3 and phosphomutants single molecule organization features based on tessellation-based automatic segmentation images. For REM1.3 and phosphomutants nanodomain size distribution and the Gaussian fits are indicated. Total nanodomain area is expressed as percentage of the total PM surface. Percentage of EOS-REM1.3 molecules localizing into nanodomains, relative to all molecules observed. Localization density refers to the number of molecules observed per $\mu \mathrm{m}^{2}$ per second. Statistics were performed on at least 13 data sets per condition extracted from 3 independent experiments. Statistical differences determined by Mann-Whitney test $* \mathrm{p}<0.05, * *$ $\mathrm{p}<0.01$. 
A

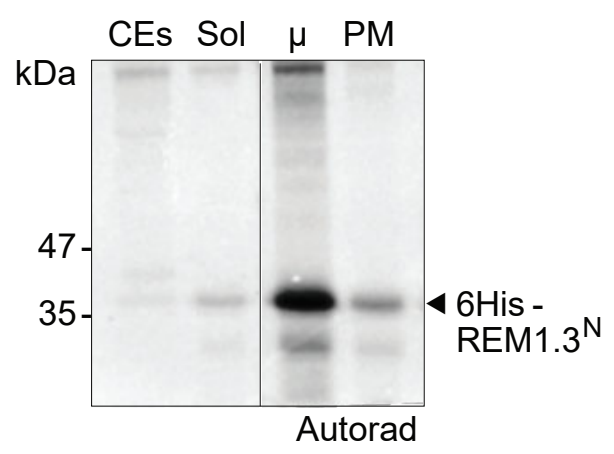

D

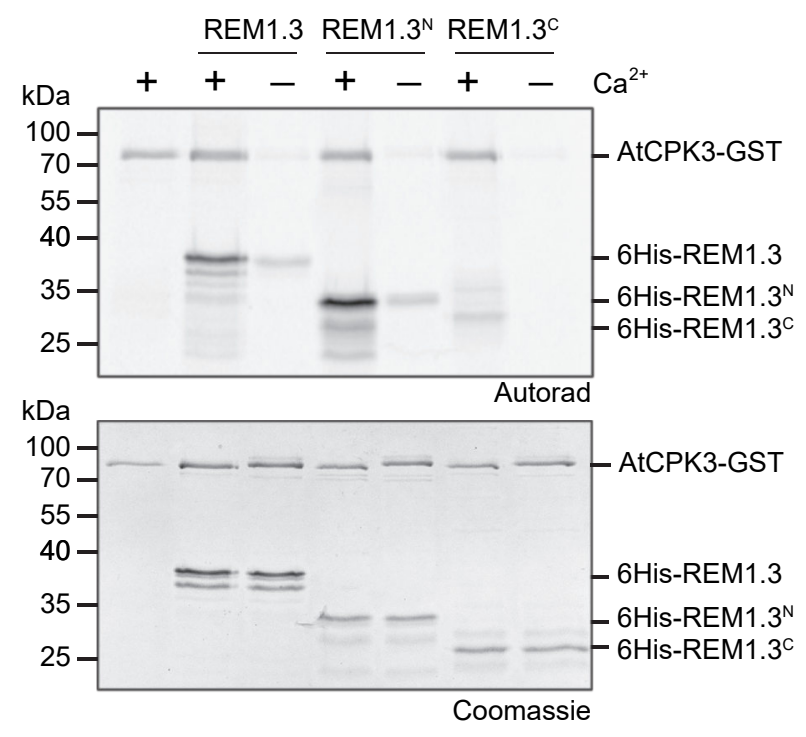

C

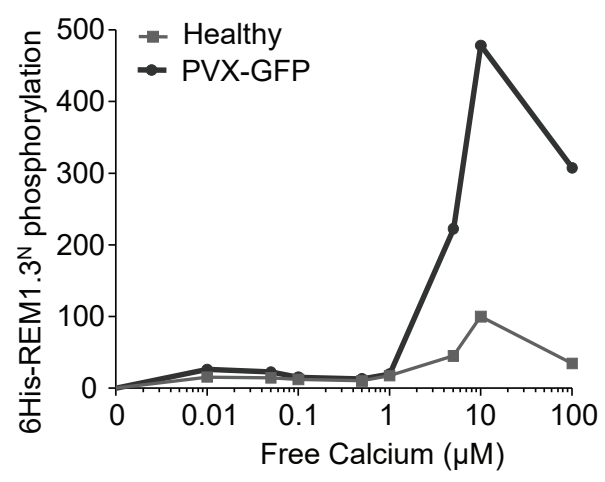

E
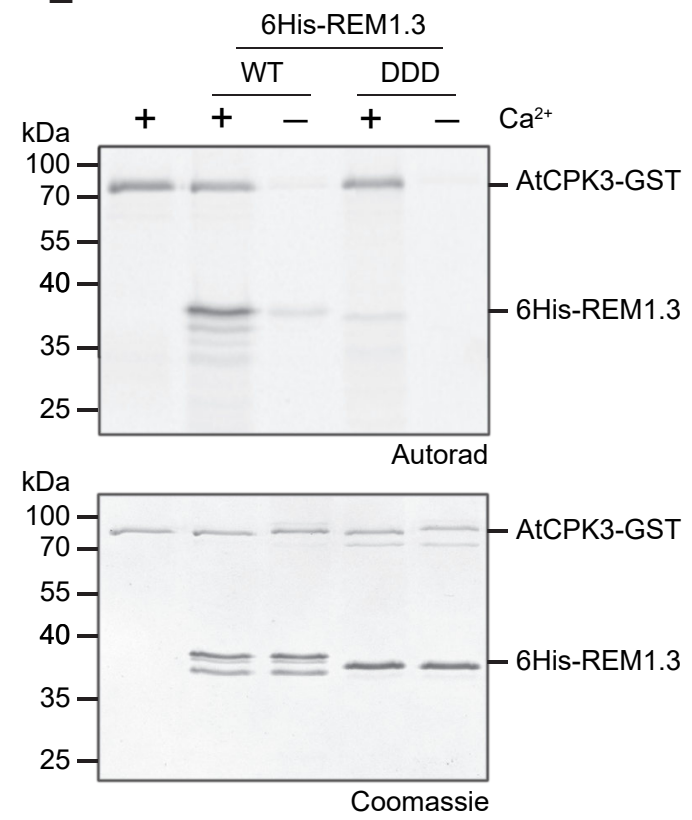

Figure 5. AtCPK3 phosphorylates REM1.3 in a calcium-dependent manner 


\section{Figure 5. AtCPK3 phosphorylates REM1.3 in a calcium-dependent manner}

(A, B) In vitro phosphorylation of purified 6 His:REM1.3 ${ }^{\mathrm{N}}$ by kinase(s) from different cellular fractions of $N$. benthamiana leaves, CEs, leaf crude extracts; Sol, Soluble fraction; $\mu$, microsomal fraction; PM, Plasma Membrane; C-PM: "Control-PM" is PM fraction not treated by TX100, but submitted to sucrose gradient; DRM, Detergent resistant membranes ${ }^{53}$. The graph represents the relative quantification of 3 independent experiments normalized to the activity in the PM fraction +/SEM.

(C) Quantification of the calcium dose response of kinase activity on 6 His-REM $1.3^{\mathrm{N}}$ phosphorylation by $N$. benthamiana microsomal extracts from healthy and PVX infected leaves.

(D, E) Autoradiography gels show in vitro phosphorylation of 6His-REM1.3, 6His-REM1. $3^{\mathrm{N}}$ and 6 His-REM $1.3^{\mathrm{C}}$ or 6 His:REM1.3 $3^{\mathrm{DDD}}$ by affinity purified GST-AtCPK3 in the presence or the absence of $\mathrm{Ca}^{2+}$. Bands corresponding to autophosphorylation of AtCPK3-GST and transphosphorylation of 6His-REM1.3 variants are indicated. Gels were stained by coomassie blue to visualize protein loading. 
A

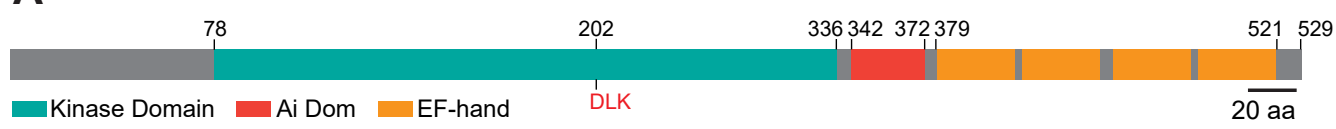

B

AtCPK3-YFP
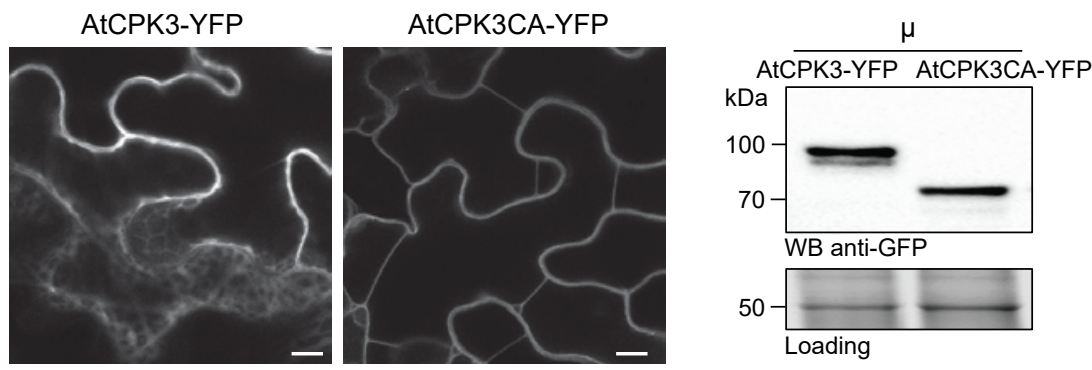

C

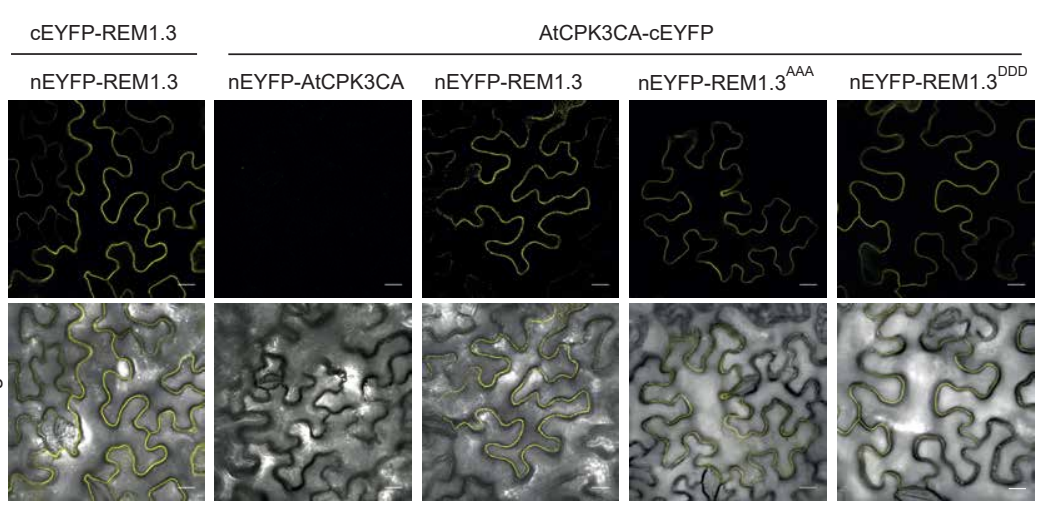

AtCPK3CA-CEYFP
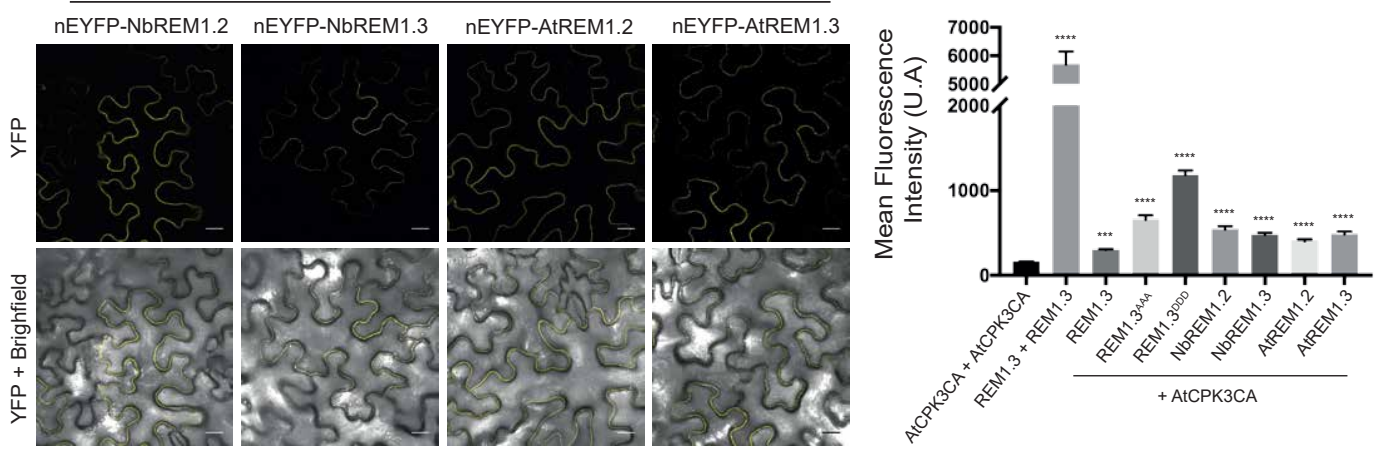

D

E
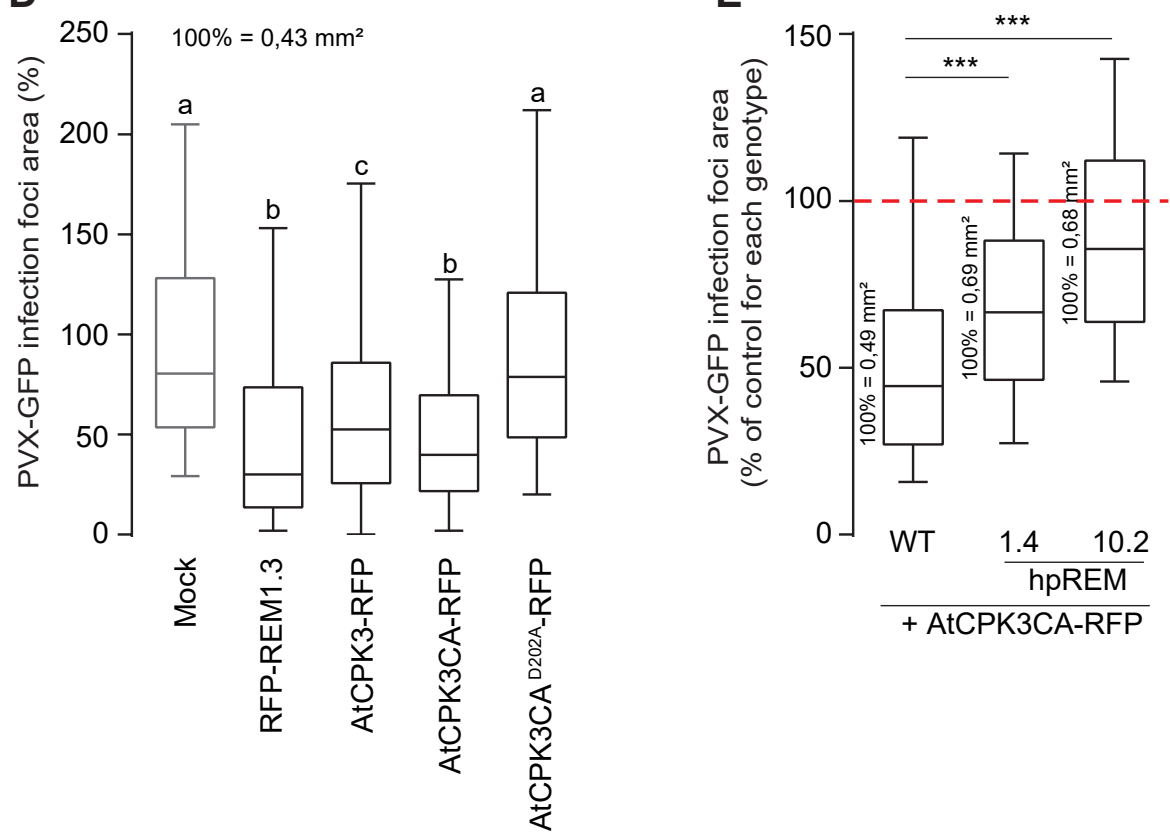

Figure 6. AtCPK3 physically interacts in vivo with group 1b REMs and impairs PVX cell-to-cell movement in a REM-dependent manner 
Figure 6. AtCPK3 physically interacts in vivo with group 1b REMs and impairs PVX cell-to-cell movement in a REM-dependent manner

(A) Primary sequence of AtCPK3. EF-hands are helix E-loop-helix F structural domains able to bind calcium. Ai : Autoinhibitory domain. The position of the DLK motif (Aspartic acid-Leucine-Lysine) at the catalytic domain conserved in all CPKs is indicated.

(B) Confocal images showing AtCPK3-YFP and AtCPK3CA-YFP localization in N. benthamiana epidermal cells. Scale bar shows $10 \mu \mathrm{m}$. Western blot against GFP showing AtCPK3-YFP and AtCPK3CA-YFP expression in the microsomal fraction $(\mu)$ of $N$. benthamiana leaves.

(C) In planta Bimolecular Fluorescence Complementation (BiFC) analysis showing interaction of AtCPK3 with Group 1 REMs. REM1.3-YFP ${ }^{\mathrm{N}} / \mathrm{REM} 1.3-\mathrm{YFP}^{\mathrm{C}}$ was used as a positive control, and AtCPK3CA-nYFP/ AtCPK3CA-cYFP as a negative control. Mean fluorescence intensity at the cell boundary level was recorded using ImageJ. Statistical differences were determined by Mann-Whitney test compared to AtCPK $3^{\mathrm{CA}}+\mathrm{AtCPK} 3^{\mathrm{CA}}$.*** $\mathrm{p}=0.0002$, **** $\mathrm{p}<0.0001$. All scale bars indicates $20 \mu \mathrm{m}$.

(D) PVX-GFP spreading in $N$. benthamiana cells expressing RFP-REM1.3 or AtCPK3 ${ }^{\mathrm{FL}}$-RFP, AtCPK3 ${ }^{\mathrm{CA}}$-RFP, AtCPK3 ${ }^{\mathrm{CA}}{ }^{\mathrm{D} 202 \mathrm{~A}}$-RFP Graph represents the area of PVX-GFP infection foci relative to the mock control (co-infiltration of PVX-GFP with empty A. tumefaciens). At least 200 PVX-GFP infection foci from at least 3 independent repeats were imaged at 5DAI. Letters indicate significant differences revealed by Dunn's multiple comparisons test $p<0.001$. .

(E) Effect of AtCPK3 ${ }^{\mathrm{CA}}$ on PVX-GFP cell-to-cell movement in WT N. benthamiana or in transgenic lines constitutively expressing hairpin REM (hpREM) constructs. At least 200 PVX-GFP infection foci from at least 3 independent repeats were imaged at 5DAI. For each $N$. benthamiana line the effect of AtCPK $3^{\mathrm{CA}}$ is expressed as a percentage of the corresponding mock control (empty Agrobacteria). Absolute values of the average foci area for each mock control are indicated. 
bioRxiv preprint doi: https://doi.org/10.1101/205765; this version posted October 19,2017 . The copyright holder for this preprint (which was not certified by peer review) is the author/funder, who has granted bioRxiv a license to display the preprint in perpetuity. It is made available under aCC-BY-NC-ND 4.0 International license.

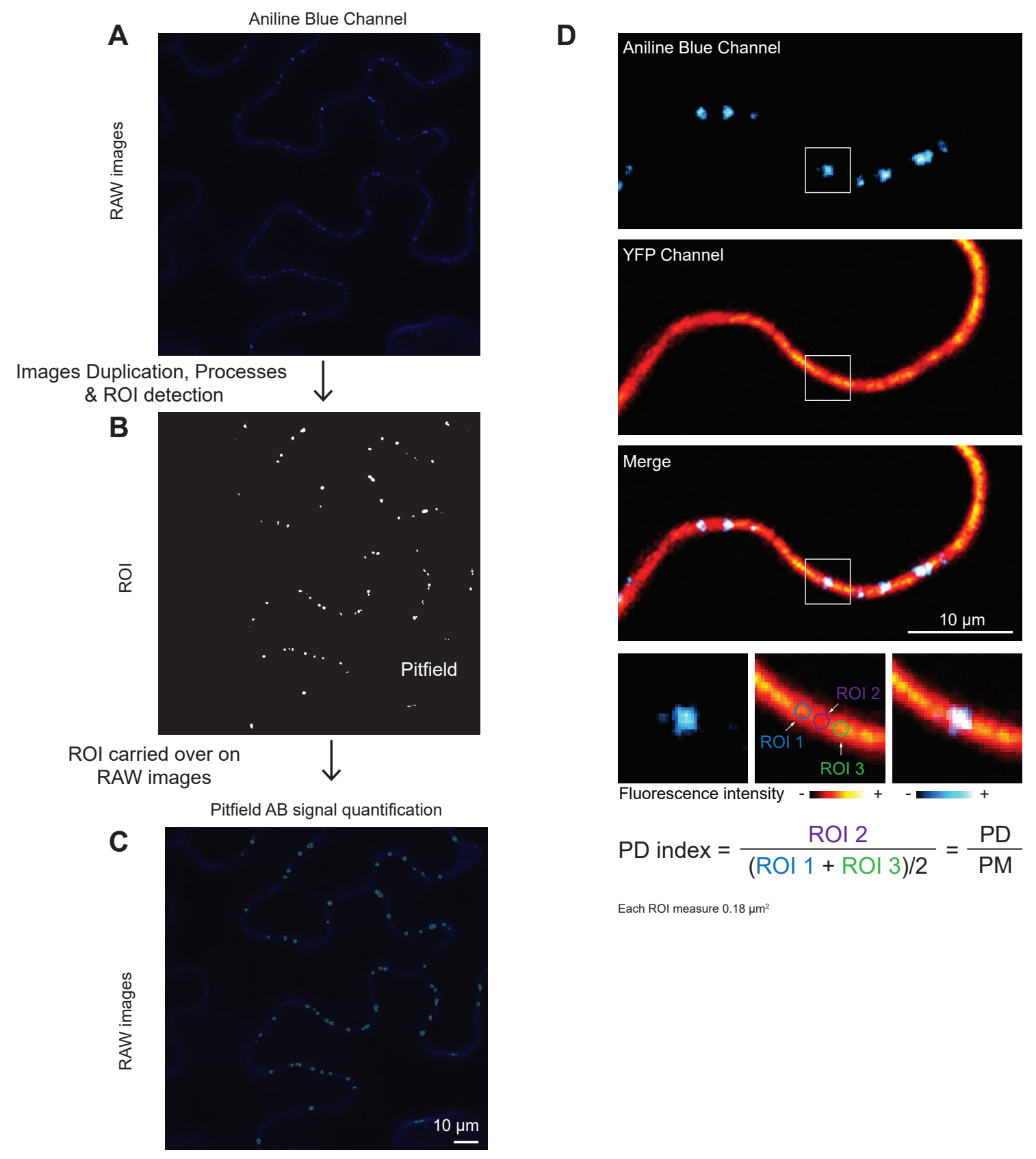

Fig EV1. Callose quantification by aniline blue staining and PD index calculation 


\section{Expanded View Figures (Supplementary figures)}

\section{Fig EV1. Callose quantification by aniline blue staining and PD index calculation}

(A) Original sample image is an 8-bit, single-channel image.

(B) Masks of total ROI objects before particle analysis were created using the following filters; background subtraction with a rolling ball radius as in ${ }^{90}$; "smooth" twice and an auto-local threshold Max Entropy dark, creating a black and white mask, used for particle detection.

(C) Overlay of outlines of the analyzed ROI (green; after particle analysis with particle size [3-100 pixel $^{2}$ circularity [0.3-1] exclude on edge) with the original image. Scale bar indicates $10 \mu \mathrm{m}$.

(D) Quantification of PD Index; after aniline blue labeled pit-field detection, YFP-REM1.3 fluorescence intensity was manually measured at pit-field level (ROI2) and surrounding PM (ROI1 and ROI3) using a circle of fixed area $\left(0.18 \mu \mathrm{m}^{2}\right)$. The PD index was then calculated as the ratio between YFP-REM1.3 pit-field fluorescence (ROI2) and the mean of YFP-REM1.3 fluorescence intensity at surrounding PM (ROI1+ROI3). 
bioRxiv preprint doi: https://doi.org/10.1101/205765; this version posted October 19, 2017. The copyright holder for this preprint (which was not certified by peer review) is the aythgrffunder, who has granted bioRxiv a license to display the pngprint in pqpqffuity. It is made

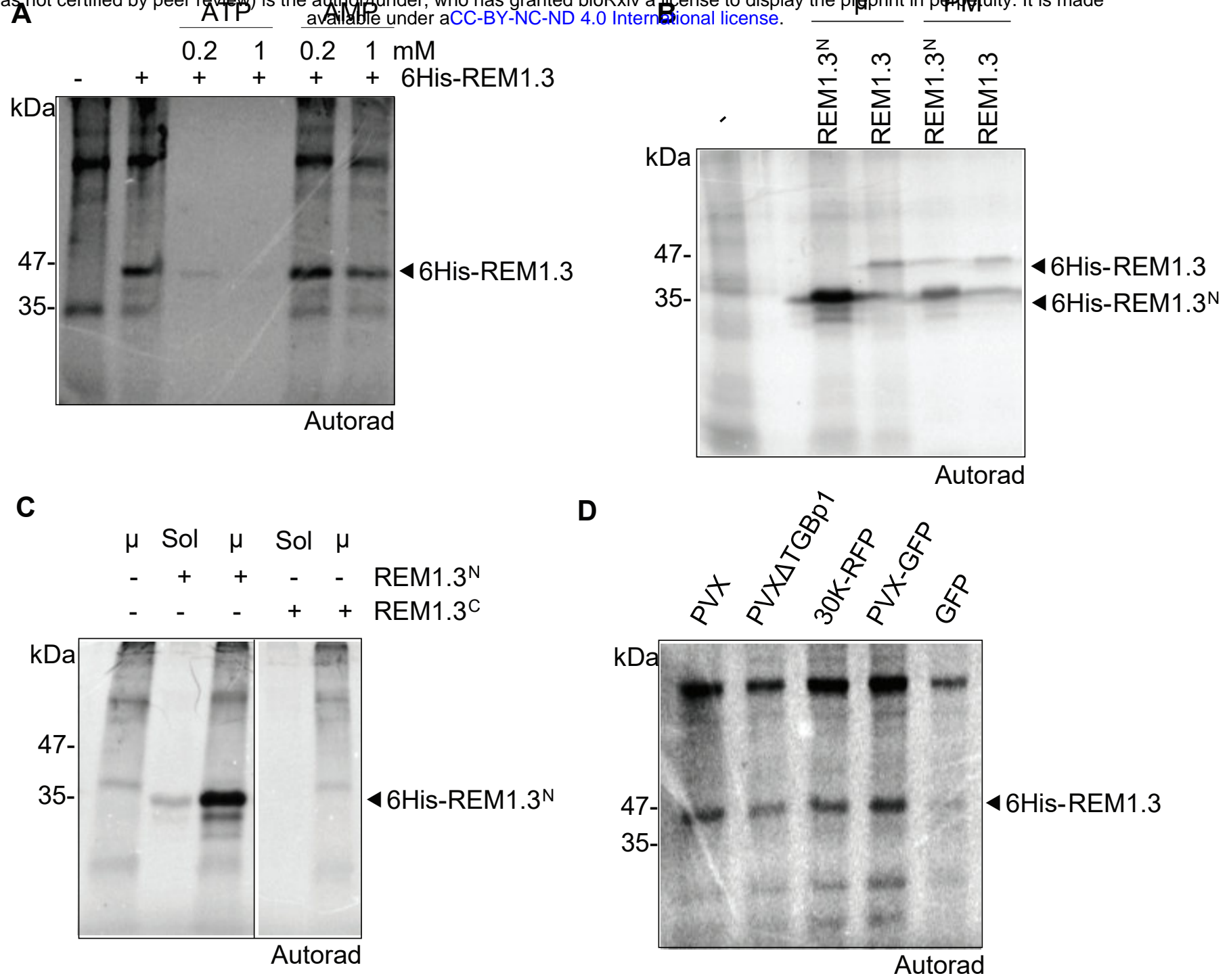

Fig EV2. Analysis of in vitro 6His-REM1.3 phosphorylation. 


\section{Fig EV2. Analysis of in vitro 6His-REM1.3 phosphorylation.}

(A) Effect of the addition of ATP or AMP in in vitro phosphorylation assays of 6His-REM1.3 by kinase $(\mathrm{s})$ in microsomal $(\mu)$ or PM extracts of $N$. benthamiana leaves developed by autoradiography.

(B) 6 His-REM $1.3^{\mathrm{N}}$ and 6 His-REM1.3 phosphorylation by healthy $N$. benthamiana leaf microsomal $(\mu)$ and plasma membrane $(\mathrm{PM})$ extracts.

(C) 6 His-REM $1.3^{\mathrm{N}}$ and 6 His-REM1.3 ${ }^{\mathrm{C}}$ phosphorylation by kinase(s) in microsomal $(\mu)$ and soluble extracts.

(D) 6His-REM1.3 was differentially phosphorylated by leaf microsomal extracts expressing the

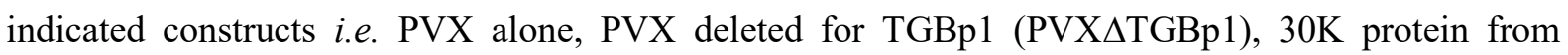
Tobacco Mosaic Virus (TMV), PVX fused to GFP, and GFP alone at 4 DAI.

In all phosphorylation experiments about $10 \mu \mathrm{g}$ of total protein extracts and $1 \mu \mathrm{g}$ of affinity purified 6 His-REM1.3, REM1.3 ${ }^{\mathrm{N}}$ or REM1.3 ${ }^{\mathrm{C}}$ were loaded per lane. 
bioRxiv preprint doi: https://doi.org/10.1101/205765; this version posted October 19,2017 . The copyright holder for this preprint (which was not certified by peer review) is the author/funder, who has granted bioRxiv a license to display the preprint in perpetuity. It is made available under aCC-BY-NC-ND 4.0 International license.

A

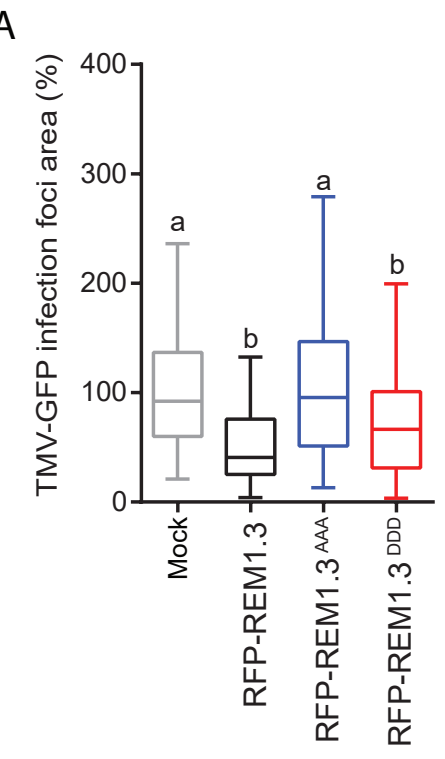

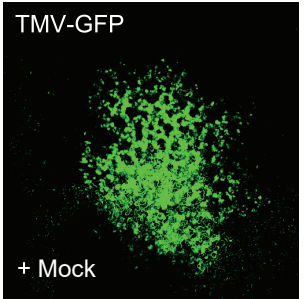

+ RFP-REM1.3 AAA

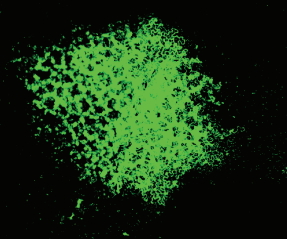

+ RFP-REM1.3

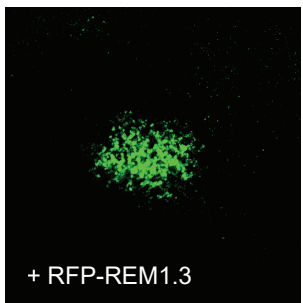

+ RFP-REM1.3 DDD

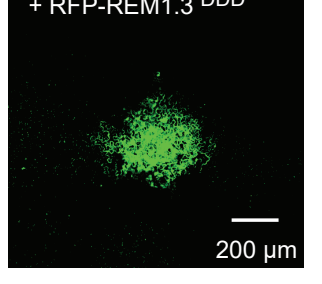

B

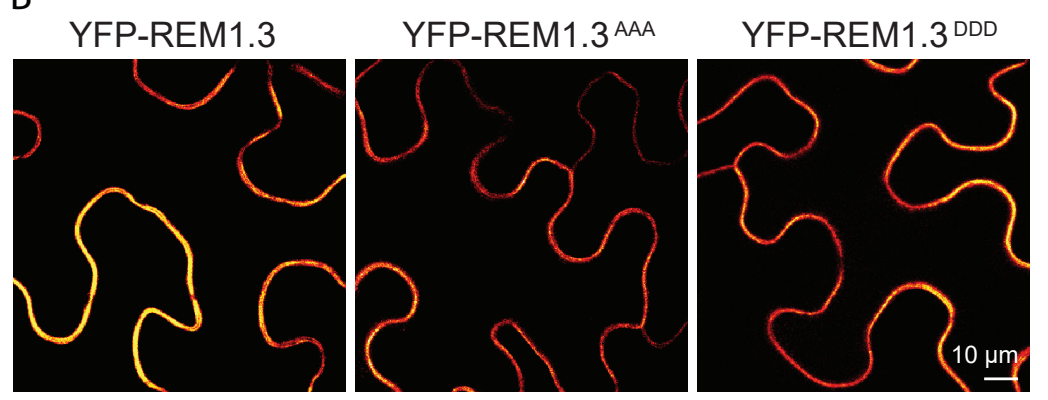

Fig EV3. REM1.3 S74 T86 S91 phosphorylation is important to regulate Tobacco mosaic virus movement and modifies the protein lateral distribution in the PM. 
Fig EV3. REM1.3 S74 T86 S91 phosphorylation is important to regulate Tobacco mosaic virus movement and modifies the protein lateral distribution in the PM.

(A) Representative epifluorescence microscopy images of Tobacco Mosaic Virus (TMV-GFP) infection foci in $N$. benthamiana leaf epidermal cells at 5 DAI. Graph represents the relative foci area of REM1.3 or phosphomutants (S74, T86 and S91 into Alanine, AAA or Aspartic Acid, DDD) compared to mock control (co-infiltration of PVX-GFP with an empty A. tumefaciens strain). About 78-128 foci per condition were measured in 2 independent biological repeats. Dunn's multiple comparison test was applied for statistical analysis, $\mathrm{p}<0.001$.

(B) Confocal microscopy images of secant views of $N$. benthamiana epidermal cells expressing YFPREM1.3, YFP-REM1.3 $3^{\mathrm{AAA}}$ and YFP-REM1.3 ${ }^{\mathrm{DDD}}$ at 2 DAI. Scale bar indicates $10 \mu \mathrm{m}$. 

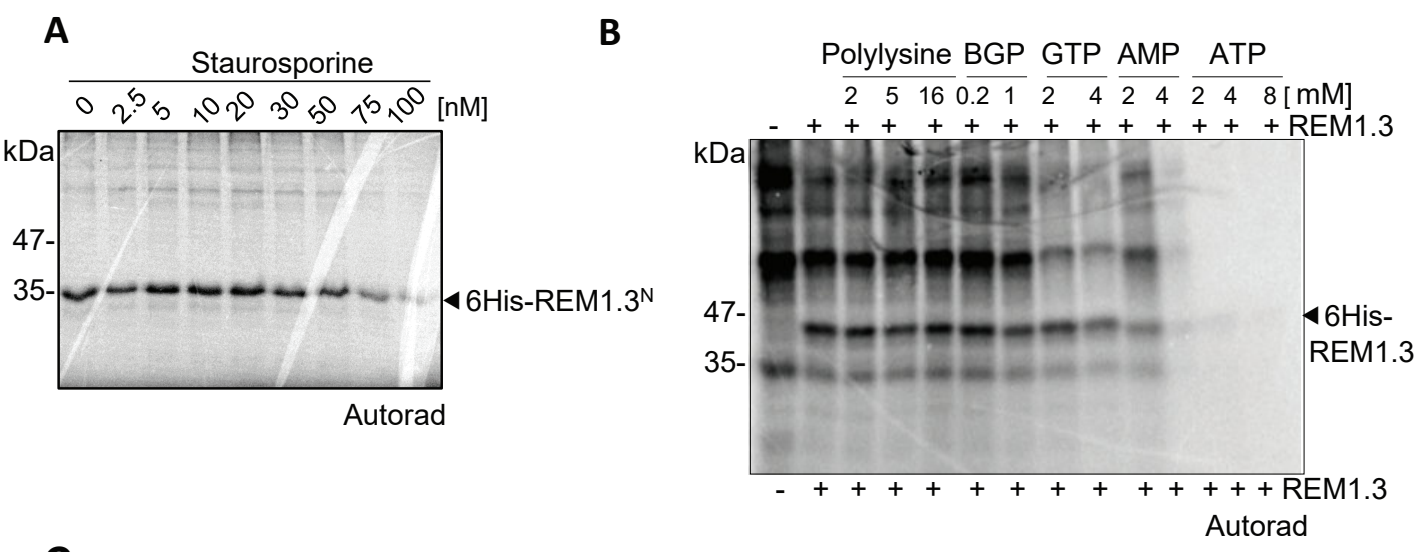

C

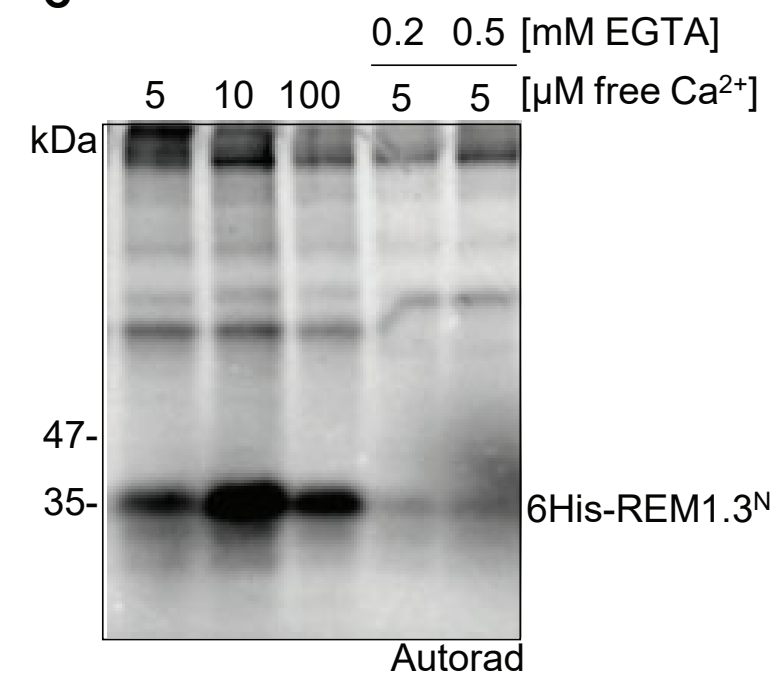

Fig EV4: In vitro characterization of REM1.3 phosphorylation conditions 
bioRxiv preprint doi: https://doi.org/10.1101/205765; this version posted October 19, 2017. The copyright holder for this preprint (which was not certified by peer review) is the author/funder, who has granted bioRxiv a license to display the preprint in perpetuity. It is made available under aCC-BY-NC-ND 4.0 International license.

\section{Fig EV4: In vitro characterization of REM1.3 phosphorylation conditions}

Autoradiography showing in vitro phosphorylation of purified 6 His-REM1.3 ${ }^{\mathrm{N}}$ (A) or 6 His-REM1.3 (B) by microsomal extracts of healthy $N$. benthamiana leaves in the presence of increasing concentrations of staurosporine (A) or Polylysine, $\beta$-glycerophosphate (BGP), GTP, AMP and ATP (B).

(C) Effect of $\mathrm{Ca}^{2+}$ and EGTA on 6 His-REM1.3 $3^{\mathrm{N}}$ phosphorylation by kinase(s) in microsomal extracts. 
bioRxiv preprint doi: https://doi.org/101101/205765; this version posted October 19, 2017 . The copyright holder for this preprint (which was not certified by peer review) is the author/funder, who has granted bioRxiv a license to display the preprint in perpetuity. It is made available under aCC-BY-NC-ND 4.0 International license.

A

StREM1.3 - MAELEAKKVE . . - IVDPAPPAPGP . . . . . . . - VEAPKEVVADEKA IVAPAL . . PPPAEEKEKPDDS NbREM1.3 - MAEVEATKVETEKVVDPTPPAPEAPAPVKEAEPVVETPKEV - ADEKA I VAPAL - - PPPEQVKEKSDDS NbREM1.2 - MAEVEATKVETEKVVDPTPPAPEAPAPVKEAEPVVETPKEV - ADEKA I VAPAL - - PPPEQVKEKSDDS AtREM1.3 MAEEQKT SKVDVESPAVLAPAKEPTPAPVEVADEK I HNPPPV $\ldots \ldots \ldots \ldots$ AtREM1.2 - MAEEQK I ALESESPAKVTTPAPADTPAPAPAE I PAPAPAPTPADVTKDVAEEKIQNPPPEQI - . - FDDS
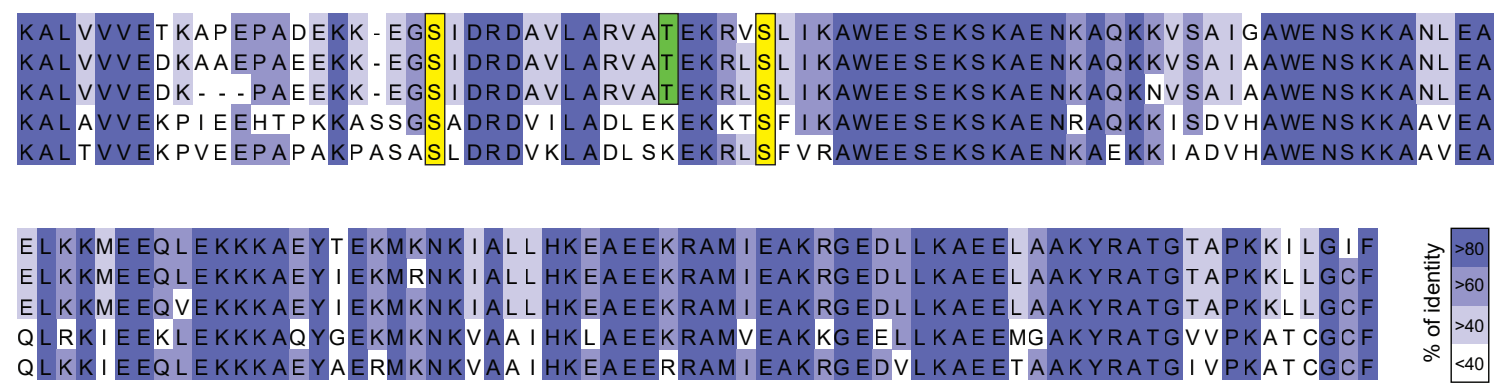

B
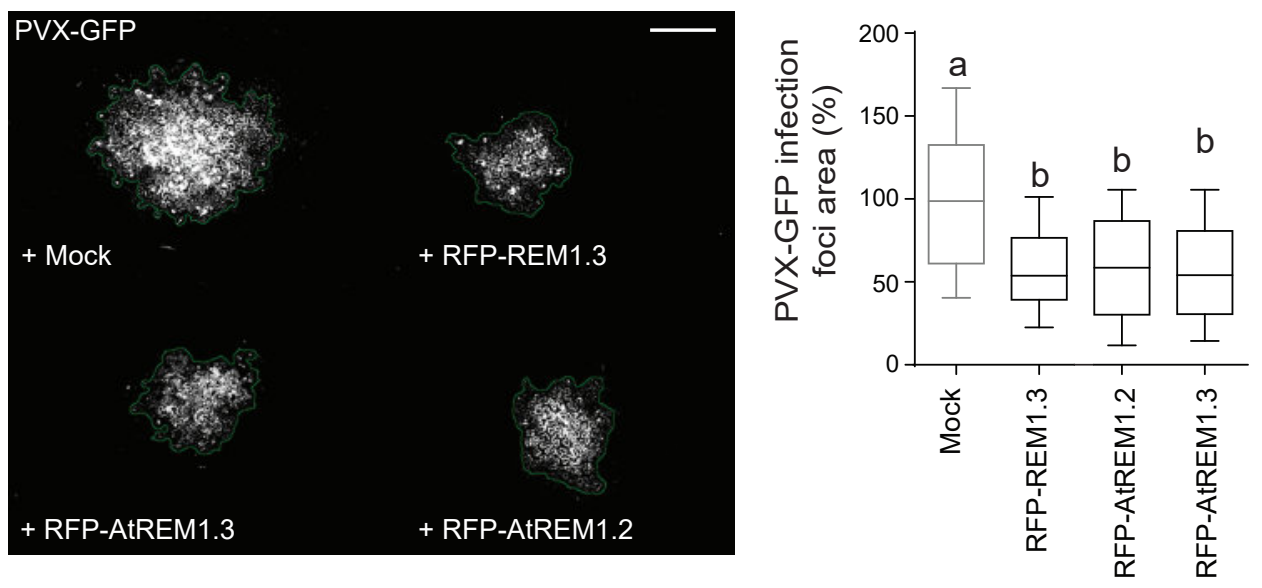

Fig EV5: Group 1b AtREMs and REM1.3 have similar behavior against PVX cell-to-cell movement in $N$. benthamiana epidermal cells. 
Fig EV5: Group 1b AtREMs and REM1.3 have similar behavior against PVX cell-to-cell movement in $N$. benthamiana epidermal cells.

(A) Clustal alignments of protein sequences of group 1b REMORINs: AtREM1.2, AtREM1.3, NbREM1.2, NbREM1.3 and REM1.3. Blue color-coding shows percentage of identity. The REM1.3 S74, T81 and S91 sites are highlighted.

(B) left, Representative epifluorescence microscopy images of PVX-GFP infection foci on $N$. benthamiana leaf epidermal cells transiently expressing RFP-REM1.3, RFP-AtREM1.2 or RFPAtREM1.3 at 5 DAI. Scale bar indicate $400 \mu \mathrm{m}$. right, Graph represents the relative PVX-GFP infection foci area in the presence of RFP-REM1.3 or Arabidopsis homologs compared to mock control (co-infiltration of PVX-GFP with empty A. tumefaciens strain). At least 184 foci per condition in 4 independent biological repeats were measured. Statistical differences are indicated by letters as revealed by Dunn's multiple comparisons test $\mathrm{p}<0.001$. 


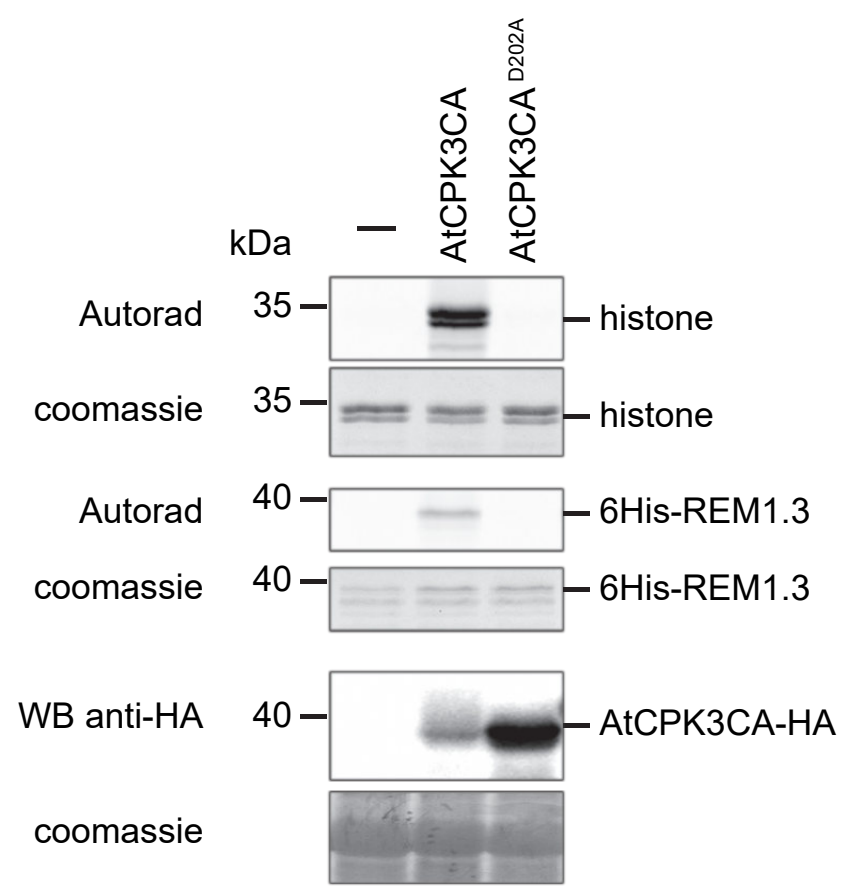

Fig EV6. AtCPK3CA ${ }^{\mathrm{D202A}}$ dead mutant does not phosphorylate REM1.3 in vitro. 


\section{Fig EV6. AtCPK3CA ${ }^{\text {D202A }}$ dead mutant does not phosphorylate REM1.3 in vitro.}

AtCPK3CA-HA and AtCPK3CA ${ }^{\mathrm{D} 202 \mathrm{~A}}$-HA were expressed in A. thaliana mesophyll protoplasts. Immunoprecipitated proteins were incubated with ATP $\left[\gamma^{33} \mathrm{P}\right]$ and submitted to an in vitro kinase assay using 6His-REM1.3 or histone as substrates. In vitro kinase assays were revealed by autoradiography. Transphosphorylation of the substrates 6His-REM1.3 or histone is indicated. Western blot against HA shows the expression levels of the expressed proteins. 

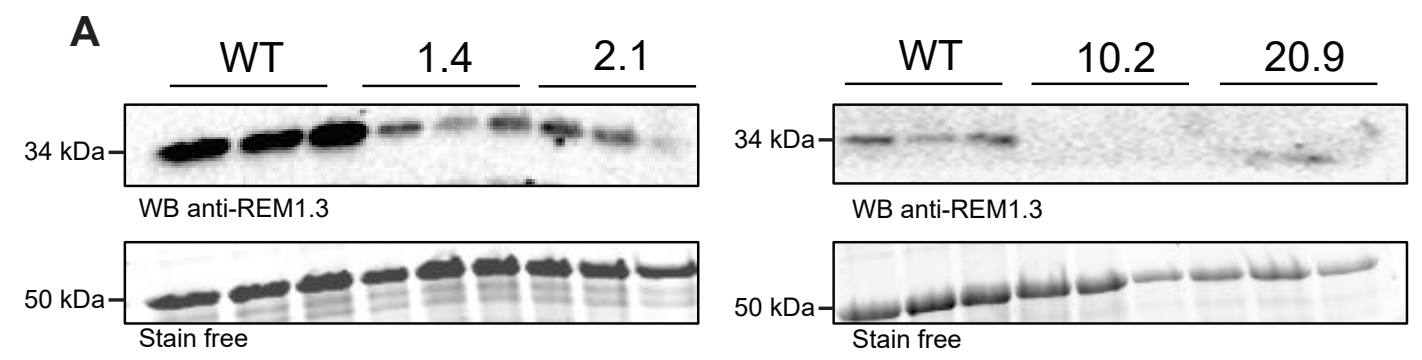

B

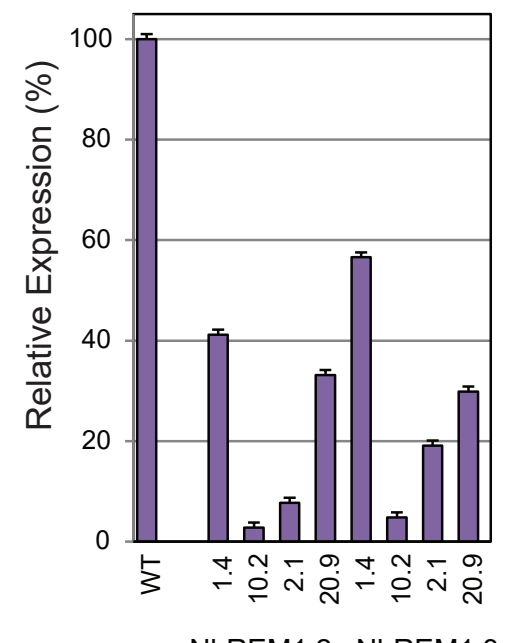

C

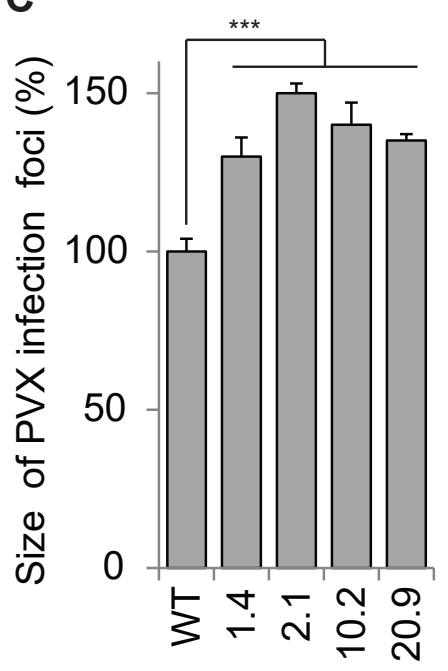

Fig EV7 Stable transgenic lines $N$. benthamiana under-expressing group1 REMORINs 


\section{Fig EV7 Stable transgenic lines $N$. benthamiana under-expressing group1 REMORINs}

(A) Protein expression levels of endogenous NbREMs in the hpREM lines, determined by Western Blot analysis using anti-REM1.3 antibodies. Protein extracts from 3 independent plants per line were used.

(B) Expression of endogenous NbREMs in the hpREM lines determined by RT-qPCR analysis. Results are expressed relative to the NbREMs expression levels in the WT background. RT-qPCR signals were normalized to actin levels.

(C) PVX-GFP spreading is accelerated in the hpREM lines. Graph represents the PVX-GFP infection foci area in the different hpREM lines compared to WT. At least 3 independent experiments were performed. Error bars show +/- SEM. Statistical differences compared to WT were determined by Mann- Whitney test $* * * \mathrm{p}<0.001$. 


\section{Materials and Methods}

\section{Plant material}

Nicotiana benthamiana plants were cultivated in controlled conditions (16 h photoperiod, $\left.25{ }^{\circ} \mathrm{C}\right)$. Proteins were transiently expressed via Agrobacterium tumefaciens-mediated transformation for virus and PD functional assays as in ${ }^{13,30}$ or for the localization experiments as described in the appendix. For subcellular localization studies and protein extractions, plants were analyzed 2 DAI or at 4DAI in the phosphorylation assays. Imaging for PVX-GFP spreading assays and plasmodesmata GFPdiffusion experiments were done at 5 DAI. Details on molecular cloning and protein work, transgenic lines generation are described in the Appendix.

\section{Viral spreading and GFP diffusion assays}

PVX-GFP cell-to-cell movement experiments were performed as previously described ${ }^{13}$, 31 , with minor modifications described in the Appendix. GFP diffusion at PD experiments was performed as previously described ${ }^{30}$.

\section{Imaging}

Live imaging and quantification were performed as described in ${ }^{31}$. For Spt-PALM, images acquisitions and processing were done as previously described ${ }^{91}$. PD index was calculated as in ${ }^{25}$. More details described in the Appendix.

\section{In vitro kinase assays}

For the in vitro REM1.3 phosphorylation assays about $2 \mu \mathrm{g}$ of total plant extracts were incubated with $1 \mu \mathrm{g}$ of affinity-purified 6His:REM1.3 protein variants for 10 minutes at room temperature and in a phosphorylation buffer (Tris-HCl 30mM, EDTA $5 \mathrm{mM}, \mathrm{MgCl}_{2} 15 \mathrm{mM}$, DTT $1 \mathrm{mM}, \mathrm{Na}_{3} \mathrm{VO}_{4} 2,5 \mathrm{mM}$, $\mathrm{NaF} 10 \mathrm{mM}$ and $10 \mu \mathrm{Ci} /$ reaction ATP $\left[\gamma^{3}{ }^{33} \mathrm{P}\right]-(3000 \mathrm{Ci} / \mathrm{mmol}$, Perkinelmer)). The buffer contained also $10^{-5} \mathrm{M}$ or gradual concentrations of free $\mathrm{Ca}^{2+}$ as in ${ }^{92}$. Reactions were performed for 15 minutes in a volume of $25 \mu$. The reactions were stopped by the addition of $15 \mu 1$ of $6 x$ Laemmli buffer. Proteins were separated by SDS/PAGE and phosphorylation status of REM1.3 was analysed by autoradiography using a phosphor-Imager and quantified by ImageQuant TL program. AtCPK3 variants phosphorylation assays were performed as described in ${ }^{93}$. 


\section{Appendix Supplementary Methods}

\section{Cloning and molecular constructs}

All vectors constructs were generated using classical Gateway ${ }^{\circledR}$ cloning strategies (www.lifetechnologies.com), pDONR211 and pDONR207 as entry vectors, and pK7WGY2, pK7YWG2, pK7WGR2, pK7RWG2, and pGWB14 and pGWB15 as destination vectors (Miyazawa et al. 2010). The REM1.3 $3^{1-116}$, REM1.3 ${ }^{117-198}$ and REM1.3 $3^{\mathrm{S} 74 \mathrm{~A}}$ T86A S91A, REM1.3 ${ }^{\mathrm{S} 74 \mathrm{D}}$ T86D S91D were synthesized in a pUC57 vector (including the AttB sites) by Genscript (http://www.genscript.com/) and next cloned to Gateway ${ }^{\circledR}$ destination vectors. AtCPK3 $3^{\text {D202A }}$ mutant was generated by site-directed mutagenesis as previously described ${ }^{94}$ with minor modifications. For BiFC experiments, the genes of interest were cloned into pSITE-BIFC- C1nec, $-\mathrm{C} 1 \mathrm{cec},-\mathrm{N} 1$ nen, and-N1cen destination vectors ${ }^{95}$.

\section{Generation of transgenic stable hairpin REM $N$. benthamiana lines}

Leaf discs were cut from $N$. benthamiana leaves, transferred on petri plates containing culture medium (complete Murashige and Skoog medium (MS) supplemented with 30g/L saccharose, pH 5,8; phytoagar HP696 (Kalys) 5,5 g/L and the hormones: AIA 0,1 mg/L, BAP $2 \mathrm{mg} / \mathrm{L}$ ) and incubated for $48 \mathrm{~h}$ in the growth room ( $16 \mathrm{~h}$ photoperiod, $30 \mu \mathrm{mol}$ photons. $\left.\mathrm{m}^{2} \cdot \mathrm{s}^{-1}, 23{ }^{\circ} \mathrm{C}\right)$. For the transformation, the $N$. benthamiana plants disk leaves were incubated with the Agrobacterium cultures (GV3101 strain) carrying the plasmid of interest for $20 \mathrm{~min}$. The leaf samples were next placed on plates with the complete medium previously described. 48 hours later, the leaf fragments were washed 3 times with sterile water (with $0,1 \%$ Tween $_{20}$ ). The leaf fragments were next washed with MS complete medium supplemented with Timentin $(300 \mu \mathrm{g} / \mathrm{mL})$. The leaves were next placed on plates with regeneration medium (MS culture medium, as previously described, supplemented with $300 \mathrm{mg} / \mathrm{L}$ of timentin and $150 \mathrm{mg} / \mathrm{l}$ of kanamycin). The plates were next incubated in the growth room. The explants were transferred to fresh regeneration medium with a maximum periodicity of 7 days until the development of callus. The regenerated seedlings were transferred to a rooting medium (MS, sucrose $30 \mathrm{~g} / \mathrm{L}$, phytoagar 5,5 g/L, timentin $200 \mathrm{mg} / \mathrm{L}$, kanamycin $150 \mathrm{mg} / \mathrm{L}$ ). The regenerated plants (T0) were transferred to the greenhouse for growth and self-fertilization. Homozygous T2 lines carrying a single transgene insertion were selected by segregation analysis on selective Kanamycin media and used for physiological studies and phenotypic characterization. The expression or silencing levels of the YFP-REM1.3 or hpREM lines respectively was controlled by cytological, biochemical and expression analysis.

\section{Viral spreading, GFP diffusion assays}

To assess spreading of PVX-GFP in tobacco leaves, A. tumefaciens strain GV3101 carrying the constructs tested were infiltrated at a final optical density at $600 \mathrm{~nm}\left(\mathrm{OD}_{600 \mathrm{~nm}}\right)=0.2$ together with the same strain carrying the plasmid pGr208, which expresses the PVX:GFP complementary DNA harboring GFP placed under the control of a Coat protein promoter, as well as the helper plasmid 
pSoup ${ }^{96}$ at final $\mathrm{OD}_{600 \mathrm{~nm}}$ of 0.001 . Viral spreading of PVX-GFP was visualized by epifluorescence microscopy (using GFP long pass filter on a Nikon Eclipse E800 with $\mathrm{x} 4$ objective coupled to a Coolsnap HQ2 camera) at 5 DAI and the area of at least 30 of PVX-GFP infection foci was measured using Fiji software (http://www.fiji.sc/) via a homemade macro or ImageJ. The expression levels of transiently expressed constructs were confirmed by Western blot. All the experiments were repeated at least three times.

\section{Transient expression in N.benthamiana}

Four-week-old N. benthamiana greenhouse plants grown at $22-24{ }^{\circ} \mathrm{C}$ were used for Agrobacterium tumefaciens-mediated transient expression. A. tumefaciens were pre-cultured at $28{ }^{\circ} \mathrm{C}$ overnight, and used as inoculum for culture at initial $\mathrm{OD}_{600 \mathrm{~nm}}$ of 0.15 in pre-warmed media. Cultures were grown until $\mathrm{OD}_{600 \mathrm{~mm}}$ reached 0.6 to 0.8 values $(3-5 \mathrm{~h})$. Cultures were then centrifuged at $3,200 \mathrm{~g}$ for $5 \mathrm{~min}$, pellet were washed twice using water to the desired $\mathrm{OD}_{600 \mathrm{~nm}}$. Bacterial suspensions at $\mathrm{OD}_{600 \mathrm{~nm}}$ of 0.2 and 0.1 were used for subcellular localization and Spt-PALM experiments, respectively. The bacterial suspensions were inoculated using a $1-\mathrm{mL}$ syringe without a needle by gentle pressure through a $<1 \mathrm{~mm}$-hole punched on the lower epidermal surface ${ }^{97}$. Transformed plants were incubated under normal growth conditions for 2 days at $22-24{ }^{\circ} \mathrm{C}$. Transformed $N$. benthamiana leaves were analyzed 2-5 DAI depending on the experiment.

\section{Epidermal cells live imaging and quantification. Bimolecular Fluorescence Complementation}

Live imaging was performed using a Leica SP5 confocal laser scanning microscopy system (Leica, Wetzlar, Germany) equipped with Argon, DPSS and He-Ne lasers and hybrid detectors. $N$. benthamiana leaf samples were gently transferred between a glass slide and a cover slip in a drop of water. YFP and mCitrine (cYFP) fluorescence were observed with similar settings (i.e., excitation wavelengths of $488 \mathrm{~nm}$ and emission wavelengths of 490 to $550 \mathrm{~nm}$ ). In order to obtain quantitative data, experiments were performed using strictly identical confocal acquisition parameters (e.g. laser power, gain, zoom factor, resolution, and emission wavelengths reception), with detector settings optimized for low background and no pixel saturation. Pseudo-colored images were obtained using the "Red hot" look-up-table (LUT) of Fiji software (http://www.fiji.sc/). All quantifications were performed for at least 10 cells, at least two plants by condition with at least 3 independent replicates. BiFC images were taken 2DAI by confocal microscopy (Zeiss LSM 880). Quantification of fluorescent intensities was performed by ImageJ.

\section{Spt-PALM, single molecule localization and tracking}

$N$. benthamiana epidermal cells were imaged at room temperature (RT). Samples of leaves of 2-weekold plants expressing EOS constructs were mounted between a glass slide and a cover slip in a drop of water to avoid dehydration. Acquisitions were done on an inverted motorized microscope Nikon Ti 
Eclipse (Nikon France S.A.S., Champigny-sur-Marne, France) equipped with a 100× oil-immersion PL-APO objective (NA = 1.49), a TIRF arm, a Perfect Focus System (PFS), allowing long acquisition in oblique illumination mode, and a sensitive Evolve EMCCD camera (Photometrics, Tucson, USA). Images acquisitions and processing were done as previously described ${ }^{91}$.

\section{In silico analysis of REM1.3 protein sequence}

Prediction of putative phosphorylation sites was performed by Diphos, DEPP and NETPHOS coupled with published data. Disordered domains were performed by pDONR VL XT.

\section{In vitro CPK3 kinase assays}

CPK3-HA was transiently expressed in mesophyll protoplasts and immunopurified with anti-HA antibodies as performed in ${ }^{93}$ while CPK3-GST recombinant protein was purified from bacterial extracts as reported in ${ }^{61}$. For in vitro kinase assays, the tagged CPK was incubated with $0.5-1 \mu \mathrm{g}$ histone or 6His-REM1.3 proteins in the following kinase reaction buffer $(20 \mathrm{mM}$ Tris $\mathrm{HCl} \mathrm{pH} 7.5,10$ $\mathrm{mM} \mathrm{MgCl} 2,1 \mathrm{mM}$ DTT, $50 \mu \mathrm{M}$ cold ATP, ATP $\left[\gamma^{-33} \mathrm{P}\right] 2 \mu \mathrm{Ci}$ per reaction, $1 \mathrm{mM} \mathrm{CaCl} 2$ or $5 \mathrm{mM}$ EGTA) in a volume of $15 \mu \mathrm{L}$ for $30 \mathrm{~min}$ at RT. The reaction was stopped with $5 \mu \mathrm{L} 4 \mathrm{X}$ Laemmli buffer, then samples were heated at $95{ }^{\circ} \mathrm{C}$ for 3 min. Proteins samples were separated by SDS-PAGE on $12 \%$ acrylamide gel. After migration, the gel was dried before exposing against a phosphorScreen to reveal radioactivity on a Storm Imaging system (GE Heathcare). The gel was then rehydrated for Coomassie staining.

\section{Protein Work}

SDS/PAGE and Western Blot analysis, protein extractions and recombinant protein purification were performed as in ${ }^{13}$. Cell fractionation and extractions followed the established protocol from ${ }^{50}$ and ${ }^{53}$.

\section{Accession Numbers}

Sequence data from this article can be found in the Arabidopsis Genome Initiative (https://www.arabidopsis.org/index.jsp), and GenBank/EMBL (https://www.ncbi.nlm.nih.gov /genbank/) databases under the following accession numbers: REM1.3 (NP_001274989), AtREM1.2 (At3g61260), AtREM1.3 (At2g45820), AtCPK3 (At4g23650). 